\title{
Urban Legend Propagation
}

\author{
Ian Dennis Miller
}

October 10, 2019

\section{Contents}

1 Introduction 3

2 Background 3

2.1 Key Terminology . . . . . . . . . . . . . . . . . . . 3

2.1.1 Serial Reproduction Task . . . . . . . . . . . . . . 3

2.1 .2 Communication . . . . . . . . . . . . . . . . . 4

2.1 .3 Networks . . . . . . . . . . . . . . . . . . . . . 4

2.1.4 Contagion and Epidemics _............ 5

2.1 .5 Memes . . . . . . . . . . . . . . . . 5

2.1.6 Social Transmission . . . . . . . . . . . . . 7

2.1 .7 Cascades . . . . . . . . . . . . . . . . 7

2.1 .8 Urban Legends . . . . . . . . . . . . . . . . . . . 8

2.2 Reviewing a study on Urban Legends . . . . . . . . . . . . . 8

2.2.1 Disgusting Stories . . . . . . . . . . . . . . 8

2.2.2 Eriksson \& Coultas Study 2: choose-to-transmit . . . . . 9

2.2.3 Eriksson \& Coultas Study 3: Receive and Transmit. . . . 11

2.3 The current work . . . . . . . . . . . . . . . . . . . 12

3 Methods 13

3.1 Computational Modelling Theory . . . . . . . . . . . . . . . 13

3.1.1 Epistemological approaches to systems modelling . . . . . 14

3.1.2 A Brief Review of Agent-Based Modelling . . . . . . . . . 15

3.1.3 Model Building and Documentation . . . . . . . . . . 17

3.1.4 Pragmatism in Individual-Based Modelling . . . . . . . . 17

3.1.5 Strong Inference . . . . . . . . . . . . . . . . . . 18

3.1.6 Pattern-oriented Modelling: A Process for Falsification . . 19

3.1 .7 Psychological Patterns . . . . . . . . . . . . . . . . 20

3.2 The Current Work . . . . . . . . . . . . . . . . . . 20

3.2.1 Study 1: Replicating Urban Legend Cascades . . . . . . . 21

3.2 .2 Study 2: Scaling Up . . . . . . . . . . . . . . . . 22

3.2.3 Study 3: Social Network Topology . . . . . . . . . . . . . 23

3.3 ODD Protocol . . . . . . . . . . . . . . . . . . 24

3.3.1 Overview . . . . . . . . . . . . . . . . . 24 
3.3.2 Design Concepts . . . . . . . . . . . . . . . . . 27

3.3 .3 Details . . . . . . . . . . . . . . . . . . . . . . 28

3.4 Simulations . . . . . . . . . . . . . . . . . . . . . . . 31

3.4 .1 NetLogo . . . . . . . . . . . . . . . . . . . 31

3.4 .2 BehaviorSpace . . . . . . . . . . . . . . 31

4 Results $\quad 31$

4.1 Study 1: Replication . . . . . . . . . . . . . . . . . 31

4.1.1 Calibrate share_threshold parameter . . . . . . . . . 32

4.1.2 Simulation Results . . . . . . . . . . . . . . . . . . 33

4.1.3 Compare choose-to-receive null model . . . . . . . . . . . 34

4.1.4 Compare choose-to-transmit null model . . . . . . . . . . 35

4.1.5 Compare both null models to empirical model . . . . . . . 36

4.2 Study 2: Receive-transmit pattern at 10x scale . . . . . . . 37

4.3 Study 3: Preferential Attachment . . . . . . . . . . . . . 38

4.3.1 Reporting Total Stories versus Average Stories . . . . . . 38

4.3.2 Simulation Results . . . . . . . . . . . . . . . . . . 39

4.3.3 Null model with Preferential Attachment topology . . . . 40

4.4 Summary . . . . . . . . . . . . . . . . . . . . 40

5 Discussion 41

5.1 The Lab and The Simulation . . . . . . . . . . . . . . 41

5.2 Scaling a serial reproduction task . . . . . . . . . . . . 41

5.3 Explanations for Emergent Network Behaviour . . . . . . . . . 41

5.4 Messages and Topologies . . . . . . . . . . . . . . . . . . 42

5.5 Error Bars and Parallel Simulations _ . . . . . . . . . . . . . 42

5.6 Statistical Inference . . . . . . . . . . . . . . . . . . 43

5.7 A General Method for Psychological Research . . . . . . . . . . . 44

6 Conclusion 44

6.1 Adapting Linear Models to Agent-Based Modelling . . . . . . . . 44

6.2 Urban Legends Require Networks . . . . . . . . . . . . . . . . . 45

$\begin{array}{ll}\text { References } & 46\end{array}$ 
Urban Legend Propagation

\section{Introduction}

This chapter is an investigation of Urban Legend propagation and the computational modelling of psychological phenomena. An urban legend is an apocryphal tale that the storyteller claims is true even though it might not be. Urban legends are a type of meme that can be passed from person to person by word of mouth.

The work begins with a finding from the literature: Urban legends propagate farther when they are disgusting (Eriksson \& Coultas, 2014). This is an example of a phenomenon known as emotional selection (e.g., Heath et al., 2001), in which the emotion evoked by a message can influence how the message is treated. The current work is a conceptual replication of urban legend transmission based on a series of studies conducted in Eriksson \& Coultas (2014), which we conducted as a computational simulation. We extended their empirical models to generalize beyond the laboratory in an effort to achieve better ecological validity.

This work offers two primary contributions. The first contribution pertains directly to the emotional selection of urban legends, which will be explored in a social network context. The second and more fundamental contribution pertains to the science of computational modelling for social psychological phenomena. While the investigation of urban legends is of interest in the context of memes and social networks, the computational modelling developments are intended to apply generally throughout psychological science.

\section{Background}

\section{$2.1 \quad$ Key Terminology}

This work builds upon the serial reproduction task, which may be used to observe story-passing behaviour in a laboratory setting (see next section, Serial Reproduction Task). The key difference in our study was the use of a different network topology, replacing the serial reproduction task with one that more closely resembled an online social network. Our model employed an epidemiological contagion mechanism which caused urban legend stories to diffuse through this network like the viral spread of diseases.

\subsubsection{Serial Reproduction Task}

This work extends the Serial Reproduction Task, developed in Bartlett (1932) for the psychological examination of memory and message passing. ${ }^{1}$ The Serial

\footnotetext{
${ }^{1}$ In this work, Bartlett also conducted a study of images, which has renewed relevance to online phenomena in modern times. The current work does not build on this pioneering experimentation - but future work ought to.
} 
Reproduction Task, which was the basis for Eriksson and Coultas' study, functions like a kid's game called "telephone operator," in which children line up and whisper a message to one another along the line. The message propagates, one child at a time; child A tells child B, who tells child C, and so on. Usually, by the end of the game, the starting message has become mutated and distorted beyond recognition.

A Serial Reproduction Task can be conducted using paper or another medium offering greater transmission fidelity than whispering. Although it is easily illustrated by a child's game, The Serial Reproduction Task is a formal research method for studying word of mouth phenomena with rigorous scientific controls.

\subsubsection{Communication}

Eriksson and Coultas described behaviour in the serial reproduction task using terms from early research by Bell Telephone on their communication network (Shannon, 1948). In this framework, there is a transmitter and a receiver, the channel that connects them, and the message that travels along that channel. Shannon (1948) includes a noise source, which manifests in the children's telephone game as interference that produces unexpected results. Shannon's work is also the gateway to information theory (e.g., Jaynes, 1957), which can serve to characterize the amount of signal that is lost or recovered in terms of a quantifiable informational property.

\subsubsection{Networks}

Graph Theory is the branch of discrete mathematics that deals with networks, which is suitable for describing the telephone networks of the 1900s and the online social networks of the 2000s - and, for that matter, the bridges of Koenigsberg in the 1700s (Euler, 1953). The serial reproduction task is an example of a very simple communication network. Graph theory provides the vocabulary ${ }^{2}$ for describing exactly how a serial reproduction experiment is topologically laid out.

In graphical terms, the serial reproduction task consists of a graph that is directed, and acyclic; "directed," meaning all the graph edges feed forward in a specific direction; and "acyclic," meaning there are no cycles or loops. Every person is represented as a node or vertex in the graph and every connection to another node is called an edge. In Bartlett's Serial Reproduction task, for each node there is only one other node that transmits a message and there is only one recipient; thus nodes have 2 edges, which is referred to as a degree of 2 . As this is a directed graph, in which the edges point in a specific direction, we say that these nodes have an in-degree of one and an out-degree of one.

\footnotetext{
${ }^{2}$ For completeness, the term plot is not synonymous with graph, despite the colloquial interchangeability of the terms. Throughout this dissertation, a plot refers to a data visualization, which is typically laid out on a euclidean plane, whereas a graph refers to a network, which itself may be plotted for the purpose of visualization. Irrespective of whether the graph is plotted, the mathematical data structure that represents a network is known as a graph.
} 


\subsubsection{Contagion and Epidemics}

Simple contagion ${ }^{3}$ is a process that involuntarily results in transmission between nodes by mere contact, alone (Le Bon, 1895). A contagious process does not involve discretion, decision making, or choices; it operates automatically like an epidemic (e.g., Goffman \& Newill, 1964). Practically nobody chooses to become sick with influenza; to the contrary, most people will make an effort to avoid contracting the flu (e.g., Butler, 2009). This type of pathogenic contagion is studied in the field of epidemiology, which has produced a robust literature on the diffusion of epidemics (e.g., Kermack \& McKendrick, 1927; Hethcote, 1974, 1994). Epidemiological modelling can be generalized beyond contagion to include processes that include imitation, choices, and decisions (e.g., Rogers, 1962 ) or, indeed, "whatever is to be diffused" (Granovetter, 1973).

Other forms of contagion also exist. Emotional contagion occurs when person A "catches" the emotions of person B (e.g., Hatfield et al., 1993). Fear spreads through social animal groups, not because the animals simultaneously choose to feel fear but through the process of contagion. Emotional contagion lies at the heart of more sophisticated social processes like sympathy, perspective-taking, and altruism (De Waal, 2008).

Social contagion occurs when person A "catches" the beliefs of person B due, again, to a process other than choice. A classic example of social contagion comes from Asch (1955), who displayed lines of varying lengths on a screen and induced participants to produce incorrect answers on the basis of group pressure. Roediger et al. (2001) induced false memories, also through social influence, when confederates systematically fed incorrect answers to participants. In these examples, ideas are transmitted socially - and, at least in these cases, it is only by willfully resisting these pressures that misinformation is not permitted to propagate.

\subsubsection{Memes}

Memes, introduced in the 7th chapter of Dawkins (1976), offer a useful theory for characterizing the messages passed through a communications network. Dawkins initially regarded memes and genes as being fairly analogous. The idea of memes has been interpreted both more radically (Blackmore, 1999) and more stylistically (Dennett, 1990; Dawkins, 1993).

A meme is a unit of culture with aspects from media studies and psychology, necessarily incorporating the human response to the meme within the system. A meme is an idea that can be repeated. In contrast with the definition used in Chapter 3 of this dissertation, a meme doesn't need to be a text; it could be anything from a musical jingle to a stone tools (e.g., Boyd et al., 2013). A meme can be mutated and changed, even unrecognizably so. For example, poems are typically altered, either intentionally or otherwise, and this mutation can manifest by being mis-remembered or mis-communicated (Boyd \& Richerson, 2000).

\footnotetext{
${ }^{3}$ as opposed to complex contagion (Centola \& Macy, 2007)
} 


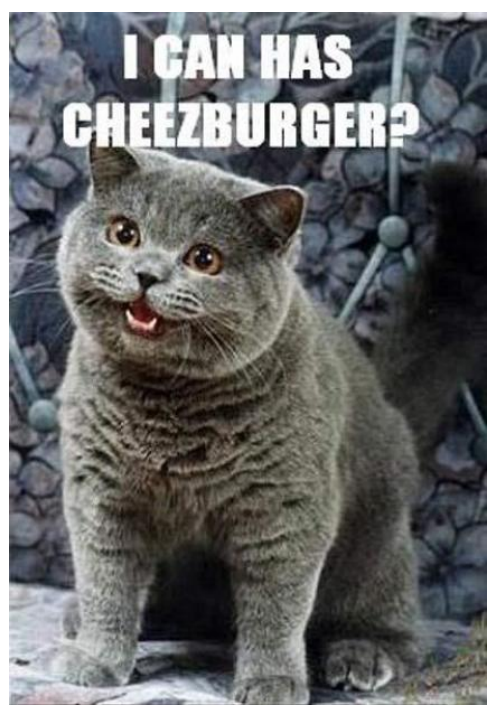

Figure 1: The "I can has cheezburger?" image macro is a historically significant image that gained a lot of attention in its time. It is an example of a LOLcat and the corrupted language was emulated and adopted by many other image macros. This style of image macro is still used in 2019, although the style is now referred to as an "advice animal."

Memes rose to prominence in light of the viral social network phenomena of the early 2000s (Blackmore, 2000). The overbearing popularity of memes raises the question of whether memes spread as a contagious process (Blackmore, 1998). According to a strict interpretation of contagion, I believe the answer is no (Hodas \& Lerman, 2014); this process more closely resembles social learning. Nevertheless, because the spread of memes does involve exposure and transmission, which are elements of contagion, the phenomenon may be subjected to epidemic analysis.

In the online context, a colloquial "meme" refers to a picture with a text overlay that is embedded within the image so that it is shared as a single digital object (e.g., Vickery, 2014). However, this particular type of meme is more accurately called an image macro (SAclopedia, 2004), which gained popularity through an early online forum called Something Awful (Kyanka, 1999). The premiere example of an image macro is the "I can has cheezburger?" cat (Nakagawa \& Unebasami, 2007), depicted in Figure 1, which produced a prolific cascade of online image sharing.

For the present purposes, the meme is the message from a media communication standpoint; it's what is transmitted and what will ultimately be the subject of the current work. 


\subsubsection{Social Transmission}

Social Transmission research incorporates individual differences and decision making in the study of message passing phenomena (Nicol, 1995; Berger \& Milkman, 2010). Social transmission, also known as word of mouth, can occur online, in-person, overheard on the street, via broadcast media, and takes many other forms (Dichter, 1966). Social transmission can even occur among nonhuman animals (e.g., Whiten et al., 2007).

Social transmission is a relaxed form of social contagion; now choice is allowed. Within this framework, messages propagate because they are interesting, popular, gratifying, persuasive, influential, and so on (e.g., Wojnicki \& Godes, 2008). The study of social transmission incorporates a variety of phenomena, including how emotions affect whether a story is transmitted or not; that is, does the message evoke strong emotions (e.g., Chattoe-Brown, 2009)? My own work with user-generated content can be classified as social transmission research (Miller, 2012).

Sharing decisions are one of the key behaviours that social transmission investigates and emotional selection is a notable factor in sharing decisions. Emotional selection occurs when a person reacts to something they read and this reaction causes the reader to behave differently with respect to the message (Heath et al., 2001). What is the likelihood to re-transmit a word of mouth message, when accounting for the emotion that the message evokes?

\subsubsection{Cascades}

Social transmission processes, when viewed from the perspective of the content rather than the individual, are called cascades (Lohmann, 1994; Bikhchandani et al., 1998). Cascades appear as a timeline or lifespan of a media object as it is passed from individual to individual (Fowler \& Christakis, 2010). This cascade may consist of a series of actions taken by multiple people or institutions, as in the case of financial cascades (Tedeschi et al., 2012). The actions related to a cascade may be simple, like clicking a share button, or they may potentially be complex.

There are countless examples of information cascades, including rumors (Allport \& Postman, 1946; Friggeri et al., 2014) and viral media (e.g., BBC Staff, 2006). We have already introduced an early image macro known as "I can has cheezburger?" demonstrating that cascades can describe multiple formats of media objects. Cascades also exist in offline contexts and have been around much longer than the Internet. Cascades can be political in nature, such as the messaging on Twitter that was seen as instigating the Arab Spring (Lotan et al., 2011). Cascades can be massive, involving billions of individuals in the case of online viral videos (e.g., Jiang et al., 2014). Cascades could also have a strong behavioural component (Leskovec et al., 2007), potentially requiring significant action from participants in the cascade (Cheng et al., 2018). 


\subsubsection{Urban Legends}

People have been telling stories for a long time - and those stories are propagated through social transmission dynamics (Edwards \& Middleton, 1990). Urban legend cascades can exist entirely apart from online media, like in the case of chain letters, mythology, and folk tales (VanArsdale, 1998). An urban legend is an apocryphal story, often with unknown origins, that is passed via word of mouth (Mullen, 1972). Frequently, the legend includes a moral or a fitnessrelated safety message.

Numerous examples of widespread storytelling crazes can be found in the literature (e.g., Stubbersfield et al., 2015). The Phantom Anesthetist is a suspicion cascade that was documented in rural America in the 1940s (Johnson, 1945). Another "mass hysteria" cascade was contagious laughter, which was treated as a health risk in some regions of the US, in part because it was viewed in the strict contagious sense (Freedman \& Perlick, 1979). A more modern urban legend is the story of the tainted Halloween candy, although the literature suggests there is little basis for the story (Best \& Horiuchi, 1985).

The phenomenon of urban legends and folklore does not particularly resemble the serial reproduction model; the nature of storytelling is usually not one-to-one (Dundes, 1965). Instead, stories are often told to groups of people, perhaps even in a broadcast context (e.g., Heyer, 2003). In the so-called "real world," urban legends don't have the harsh experimental constraints that a serial reproduction task imposes.

An urban legend can be thought of as a meme and the subtle variations of the story may be viewed as mutations. The story could be described as an information cascade or it could be framed in terms of individual sharing episodes. Emotional selection factors can influence urban legend propagation, including the details that are emphasized and those that are remembered by listeners.

\subsection{Reviewing a study on Urban Legends}

Having reviewed some of the literature, we now turn to Eriksson \& Coultas (2014) for its methodologically refined investigation of urban legend phenomena. Their work will be dissected so it can be "reverse engineered" to construct a computational replication based upon it. In many ways, the current work is a computational extension of Eriksson \& Coultas (2014).

\subsubsection{Disgusting Stories}

Eriksson \& Coultas (2014) studied disgust as a moderator for urban legend sharing. ${ }^{4}$ I was particularly drawn to their methodological separation of the

\footnotetext{
${ }^{4}$ As an aside, I was going to conduct a similar study in 2013, although with humor instead of disgust. At the time, I had just finished my master's thesis (Miller, 2012) and I found that I really wanted to dissect the process of meme transmission. Thanks to Eriksson \& Coultas (2014), I didn't have to do that work; the results they produced are just as useful to me. I love this article and they actually saved me a huge headache.
} 


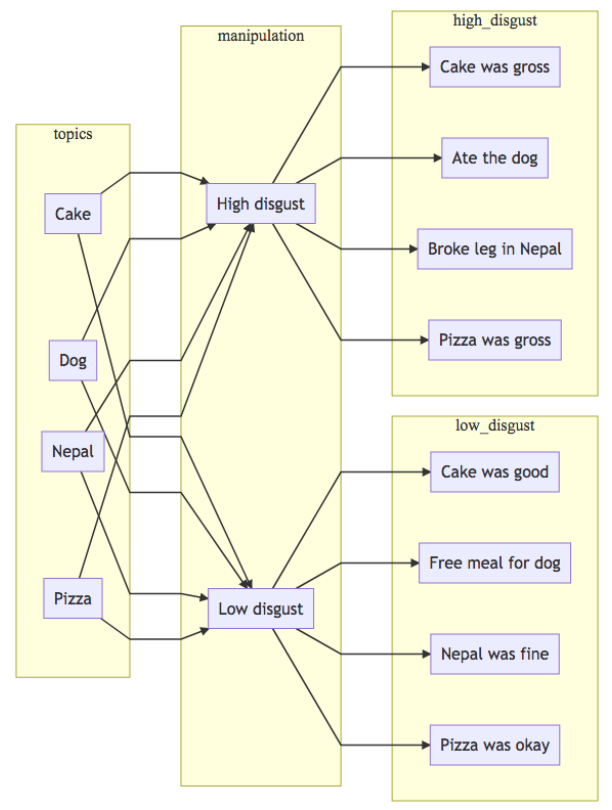

Figure 2: This diagram depicts the process by which story topics are manipulated to create high/low disgust versions.

urban legend transmission process into 3 stages that map onto Shannon's communication theory (Shannon, 1948). In the receive stage of Eriksson \& Coultas, participants chose whether or not to read an urban legend. During the encode/retrieve stage, participants engaged in a memory and recall process with urban legend stimuli. Finally, during the transmission stage, the story was actually shared to another participant.

Eriksson \& Coultas (2014) leveraged the serial reproduction task, a la Bartlett (1932), in which one participant transmits stories to the next participant, in sequence. Eriksson \& Coultas found that almost all their urban legend cascades were extinguished before the end of the serial reproduction tasks, but this effect was moderated by how disgusting the urban legends were. Low-disgust cascades ended significantly sooner than high-disgust cascades.

This finding is consistent with previous studies (e.g., Heath et al., 2001) but it is also surprising: in the real world, some urban legend cascades survive for years or centuries. The laboratory results do not appear to resemble the sort of "viral cascades" that real-world observation suggests are possible.

\subsubsection{Eriksson \& Coultas Study 2: choose-to-transmit}

In study 2, Eriksson \& Coultas investigated whether various characteristics of a story, as rated by participants, affected its transmission properties. Were disgusting stories more likely to be transmitted than their non-disgusting coun- 


\begin{tabular}{lcccrr} 
TABLE 2 & $\begin{array}{l}\text { Results of maximum likelihood estimation of pass along ratings of stories as a func- } \\
\text { tion of story manipulation and ratings of story qualities (Experiment } 2 \text { ). }\end{array}$ \\
\hline Effect & Estimate & SE & df & t value & $p$ value \\
\hline Intercept & -0.86 & 0.34 & 234 & -2.51 & 0.012 \\
Disgust version & 0.79 & 0.21 & 234 & 3.76 & $<0.001$ \\
Social version & 0.08 & 0.17 & 234 & 0.46 & 0.648 \\
Amusing & 0.22 & 0.06 & 234 & 3.84 & $<0.001$ \\
Interesting & 0.35 & 0.07 & 234 & 5.03 & $<0.001$ \\
Plausible & 0.25 & 0.05 & 234 & 4.88 & $<0.001$ \\
Surprising & 0.18 & 0.06 & 234 & 2.92 & 0.004 \\
& & & & &
\end{tabular}

Disgust version is coded 1 for high, o for low; similarly, social version is coded 1 for social, o for asocial. Because each participant rated four stories, the model included an individual-specific error term to account for individual idiosyncracies in pass along ratings.

Figure 3: Eriksson \& Coultas (2014); p. 14

terparts? And further to that question, would the disgust level of a story affect ratings of how funny the story was?

Methods Eriksson \& Coultas generated four story topics: 1) cake; 2) dog; 3) Nepal; and 4) pizza. Each topic was converted into a high and low disgust variation, as depicted in Figure 2. In the low-disgust version of the dog topic, a dog received an unexpectedly delicious meal from a restaurant. ${ }^{5}$ In the highdisgust version, the restaurant cooked the dog and served it to its owner.

The high-disgust version of the dog story violates numerous expectations about purity and morality that are associated with appraisals of disgust (Schnall et al., 2008). These four base stories were then presented to 80 Mechanical Turk participants who were randomly assigned to receive stories manipulated by disgust level. Participants rated the stories along several dimensions, including humor, disgust, and the likelihood they would pass along the story. Pass-along ratings corresponded to the transmit phase of communication and may be interpreted as "intention to transmit."

Results Eriksson \& Coultas found a positive effect of disgust upon transmission intentions, which replicates earlier findings that disgusting stories are prolific in online communities (Heath et al., 2001). In fact, several of the measured dimensions, including humor, were related to transmission, which can also be interpreted as a replication of Berger (2011).

One of the principle results of this study is a linear model of urban legend transmission. Figure 3, which is quoted directly from the article, contains the estimates from their linear model. These maximum likelihood estimates were interpreted as slopes corresponding to the effects of disgust, humor, and so on. This model tells me that on average, most stories are not shared because the

${ }^{5}$ Eriksson \& Coultas were aware that this manipulation does not strictly involve disgust, alone. In this example, the story is not merely benign but it is also humorous. 


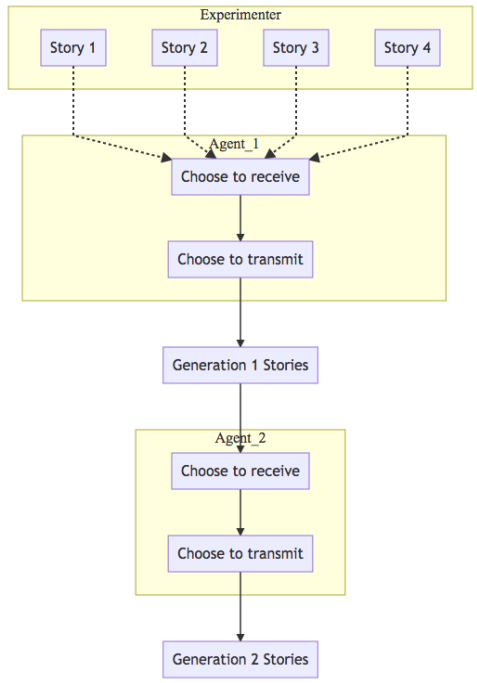

Figure 4: This flowchart depicts the research method used in Eriksson \& Coultas Study 3. The experimenter delivers 4 stories to the first participant. That participant chooses whether to read any, and subsequently, whether to share any. The stories are then passed to the next participant and the process is repeated.

intercept is nearly a standard deviation below zero. However, disgusting stories counteracted the general bias against sharing, such that disgusting stories were transmitted at a significantly higher rate. The estimates in Figure 3 were used as parameters for the current work's computational model.

\subsubsection{Eriksson \& Coultas Study 3: Receive and Transmit}

Study 3 from Eriksson \& Coultas (2014) focused only on the receive and transmit stages of the serial reproduction task. 80 participants were grouped into pairs that were organized into 40 separate serial reproduction tasks. Within each serial reproduction task, participants were assigned a sequence number indicating their order of action. The participant who acted first was referred to as the Generation 1 participant and the one who acted next was Generation 2.

Generation 1 participants were presented with four stories that were written on paper. Participants could choose whether to read each story and then choose whether to transmit them to the next participant. While each story had the chance to live for 2 generations - a cascade of length 2 - the full cascade could only occur when both participants decided to transmit those stories. In practice, most story cascades resulted in a length shorter than 2 .

Within each generation, there are 2 decision steps involving an interaction between the participant and the content. During the receive step, participants looked at the titles of the stories they were given and decided whether to read 


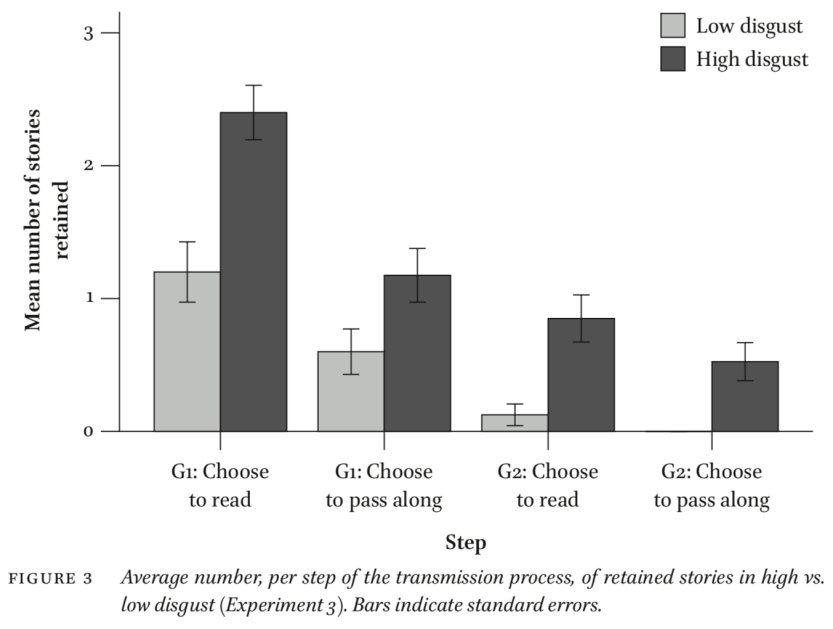

Figure 5: Eriksson \& Coultas (2014); p. 17

each story. To complete the receive step, participants actually read the stories they wanted to read. Then, during the transmit step, participants decided whether to share any stories. To complete the transmit step, experimenters collected the stories that were shared and provided them to the next person in the task.

Unlike the previous study, which measured intention to share, in this study participants actually engaged in the transmission activity. This paradigm realistically simulates the filtering effects present in the propagation of urban legends. The flowchart in Figure 4 depicts person 1 receiving four stories, choosing whether to read any of them, and finally choosing to transmit the stories to person 2. Presuming person 2 received any stories, they may choose whether to read the stories they received and subsequently choose whether to transmit any.

Eriksson \& Coultas (2014) tracked the number of stories that were retained after 2 generations. The plot, reproduced in Figure 5, demonstrates that as stories pass from person to person, fewer and fewer stories are retained. Notice that high-disgust stories are retained at a higher rate than low-disgust. By the time the generation 2 participant engages in the transmission step, there are no low-disgust stories left and there are few high-disgust stories remaining. In showing different outcomes based on disgust levels, Eriksson \& Coultas (2014) demonstrated evidence for emotional selection both during the receive stage and during the transmit stage.

\subsection{The current work}

Chapter 3 involved the naturalistic observation of memes spreading on twitter, a method that was strongly grounded in the phenomenological world but which 
included no experimental manipulation. By contrast, Eriksson \& Coultas (2014) used strong experimental controls but resorted to the laboratory to accomplish it. The current work is an effort to obtain the best of both worlds, transporting the findings from the laboratory into a new environment that more closely resembles aspects of the real world.

In the experimental sciences, validity is related to the proper controls applied to the proper experimental variables - but these controls can also interfere with validity (Campbell, 1957). Ecological validity, one form of external validity, is a common and sometimes-valid criticism of laboratory studies conducted in the psychological sciences (e.g., Schmuckler, 2001). The controls afforded by laboratory research can be at odds with ecological validity but, as Campbell (1957) points out, both internal and external validity are important criteria for experimental research.

The findings reported in Eriksson \& Coultas (2014) demonstrate excellent internal validity; they have expertly dissected the urban legend storytelling episode. However, the use of the serial reproduction task is a departure from the nature of human social networks, which are rarely one-to-one, interfering with the generalizability of the results. Correspondingly, the transmission results do not appear to be consistent with the proliferation of urban legends that are observed in the world, as many urban legends persist for years or even centuries. The network itself is therefore one of the key study elements that can be changed to improve the external validity of the results.

This work considers a program of research consisting of two phases: 1) an internally-valid laboratory phase that produces a refined model; and 2) an externally-valid simulation phase that examines that model in an ecologicallygrounded environment. The current work translates the Eriksson \& Coultas (2014) urban legend transmission model into a computational modelling framework. This transmission model may then be evaluated under different network conditions that more closely resemble the real world. In contrast with the implausible findings produced by a serial reproduction task, we seek recognizable, epidemic sharing of urban legends to emerge from the very same model specified by Eriksson \& Coultas (2014).

\section{Methods}

This methods section begins with a review of the computational modelling literature, then describes how computational modelling techniques were applied in the current work.

\subsection{Computational Modelling Theory}

The computational modelling of complex systems is discussed in many disciplines, each of which has evolved a slightly different vocabulary for saying similar things. The current work uses Agent-Based Modelling (ABM), which is gaining prominence in the Computational Social Sciences community. A dif- 
ferent modelling approach called Individual-Based Modelling is better-known within the ecological modelling community.

Although these two approaches have largely converged into a single practice, they have very different theoretical roots. I initially ignored the differences between these two literatures but I later determined this was an oversight. The ecological literature provides strong theoretical foundations for modelling decisions that I now believe are essential to computational modelling in the psychological sciences. Therefore, this section begins with a synthesis of these literatures for applications in psychology.

\subsubsection{Epistemological approaches to systems modelling}

Mathematical models have been central to the scientific investigation of physical systems for millennia (e.g., Dreyer, 1906). When the principles of those models are mechanically embodied, as with astronomical computing mechanisms like the orrery and clock, those systems can be simulated and predictions can be investigated (e.g., Pope, 1804; Reid, 1832). More recently, simulation techniques have been applied to social systems, including economies (e.g., Walras, 1874), demographics (Dublin \& Lotka, 1925), and societies (Schelling, 1971).

Economics Walrasian models were an early approach to the analysis of complex human systems. Walrasian models can be characterized in terms of their formal approach that frequently involves mathematical functions that are solved in order to compute equilibria that balance the equations. The strengths inherent in early economic models derive from their formal specification, permitting analysis by mathematical methods. A classic example of the Walrasian approach is to compute the price of goods from a supply curve and a demand curve, in which a single price may be found at the intersection of these curves. Critics of equilibria models - including proponents of Agent-based Computational Economics (e.g., Tesfatsion, 2003) - highlight the following shortcomings: 1) Walrasian systems do not support recursive, micro- and macro- level analysis; and 2) the formal notation can interfere with communication. Early iterations of the current work attempted to apply a Walrasian approach to social scientific research with memes - but without much success.

Individual-Based Modelling Individual-Based Modelling takes patterns of empirical observations and builds models from them (Schank, 2001; Grimm \& Railsback, 2005). The Individual-Based Modelling approach was developed in the ecology literature, where regressions and algorithms are used to describe systems of wildlife. Some famous examples of individual-based modelling involve forests of trees (Liu \& Ashton, 1995), salmon spawning (Railsback \& Harvey, 2002), and the natural world at large. Insofar as many human behaviours are closely related to other animal behaviours - particularly to other primates - the epistemology underlying Individual-Based Modelling is a good match for social psychological research. 
Agent-Based Modelling Agent-Based Modelling (ABM) is a discrete, computationoriented modelling approach that is well-suited to social systems (e.g., Epstein \& Axtell, 1996; Epstein, 1999; Bonabeau, 2002; Macy \& Willer, 2002; Berry et al., 2002; Bankes, 2002). ABMs are sometimes represented algorithmically as finite state machines or as decision trees consisting of if-then statements. A different term for this approach is multi-agent systems (MAS), as it applies to the fields of robotics, computer science, or manufacturing (Jennings et al., 1998). ABM systems are examined by performing computations in a loop to see what inherently-unpredictable results emerge. These may not be analyzed in situ using the same formal methods that are suitable for classical economic systems.

\subsubsection{A Brief Review of Agent-Based Modelling}

Agent-Based Modelling (ABM $)^{6}$ is a method for studying complex systems including people, animals, cars, and products (Railsback \& Grimm, 2011; Wilensky \& Rand, 2015). A key characteristic of the ABM method is the use of an agent population in which every agent implies some sort of computation.

Demographics Dublin \& Lotka (1925) studied US population growth using a computational approach that might be the earliest example of ABM I've found. Dublin \& Lotka (1925) represented 100 million Americans in their simulation and, for each one, computed the consequences of mating and childbirth in order to estimate the US national population over time.

Cellular Automata A precursor to modern agent-based modelling is the computational field of cellular automata (CA), in which each automaton is an abstract unit (a cell) that performs a computation (von Neumann, 1966). John Conway's Game of Life is a classic example of cellular automata that is laid out on a grid, like a checker board, in which each square on the grid changes colours based on the colours of neighboring squares (Conway, 1976). The Game of Life produces unexpectedly-fascinating emergence, illustrating the profound and chaotic relationship between simple rules and complex outcomes. Conway (1976) elegantly demonstrates the non-trivial properties of emergence, extending far beyond mere extrapolation in order to produce unpredictable results.

Sociology Racial segregation was studied in Schelling (1971) using a computational simulation of a virtual neighborhood. The agents in Schelling (1971) were influenced by the effects of homophily: each agent measured the ratio of other-race agents in their vicinity and if a threshold were exceeded, they would move to a new location. By varying the homophily threshold, Schelling's model demonstrated that even a minor preference for homophily could manifest communities of segregation. As demonstrated by Schelling (1971), which was computed using a chess board, a computer is not a requirement for Agent-Based

${ }^{6} \mathrm{ABM}$ is also referred to as Agent-Based Social Simulation, or ABSS. 
Modelling. Schelling's computational simulation convincingly bridges the social world and the mathematical, using parsimonious rules to inspect the complex sociological phenomenon of racial segregation.

Generative Social Science The Sugarscape Model was an important AgentBased Model from which a wide range of natural phenomena were shown to emerge, including: life and death; sex, culture, conflict; disease; society; wealth; social networks; migration; warfare; trade; markets (Epstein \& Axtell, 1996). ${ }^{7}$ Epstein \& Axtell obtained these emergent outcomes through the accumulation of simple rules. ${ }^{8}$ Sugarscape demonstrated that Agent-Based Modelling is a flexible method that can be applied to complex, human social phenomena using models that are easy to describe while offering practical insights on the phenomena they are applied to.

ABM Toolkits Each of the studies described so far simultaneously developed novel theories as well as a computing mechanism for exploring them, which usually amounted to a custom software implementation built expressly for the task. By the late 1990s, scientists began to experiment with general purpose ABM toolkits to provide common modelling resources to facilitate reuse and communication. An ABM toolkit is a programming environment in which a model is described with computer software that determines how agents behave. The ABM toolkit also provides an environment within which the agents exist, which may also be controlled using computer code. Once the agents and environment are described, the ABM toolkit facilitates the simulation of the model by placing agents into the environment and executing the instructions that cause the agents to act. These simulations, implemented by the ABM toolkit, are executed in order to produce the data that is ultimately analyzed.

There are currently many ABM toolkits, including NetLogo (Wilensky, 1999), REPAST (García \& Rodríguez-Patón, 2016), and MASON (Luke et al., 2005). ${ }^{9}$ Of these toolkits, NetLogo is the most widely used across the social sciences, becoming the Lingua franca for ABM work in many fields. The ecologists and individual-based modelling community have also come to use NetLogo, despite originating from a different intellectual lineage (e.g., Swarm Development Group, 2016). From the perspectives of usability and communication, NetLogo provides many tools that are useful to researchers. ${ }^{10}$

\footnotetext{
7 The Sugarscape model has been been extremely influential to my own thinking.

8 Epstein \& Axtell (1996) is now considered part of a Generative Social Science trilogy, which includes Epstein (2006) and Epstein (2014).

9 I used REPAST to implement Miller (2012) but I found that it was difficult to keep the toolkit running over the course of several years, which interfered with reproducibility. I initially used MASON for the current chapter but, after a year, I actually started from scratch with NetLogo. MASON is extremely general-purpose, which I initially considered to be a strength but eventually this constituted a net cost because I had to write so many basic routines from scratch.

10 I've tried going my own way, developing some of this stuff from scratch, but I found that this is a dead-end because this introduces too many barriers to sharing work with other researchers. It's worth it to communicate with a community that already exists.
} 


\subsubsection{Model Building and Documentation}

The model building framework described in Grimm \& Railsback (2005) was adapted for the current work. Together with Railsback \& Grimm (2011), these procedures provide a complete workflow for conducting a computational modelling study according to the Individual-Based Modelling approach.

In order to facilitate communication among computational modelers, Grimm et al. (2006) propose a model description framework called the ODD Protocol, which stands for Overview, Design Concepts, and Details. The current work uses the ODD Protocol for documentation about its models, which will be employed a little later in this section.

Altogether, this literature provides a methodology that I have applied to the psychological science domain. I have summarized the model building method used in the current work, which was adapted from the theories of Grimm, Railsback, and others, as follows:

1. Formulate the model using the Overview, Design Concepts, and Details (ODD) protocol.

2. Identify characteristic patterns of the emergent behaviour. This corresponds closely to the Pattern Oriented Modelling process described in Railsback \& Grimm (2011).

3. Describe the criteria for pattern matching. For the current work, I am characterizing "pattern matching" as a sort of error-minimization process. Pattern matching can be conceived of in different ways - and I think that developing stronger criteria for pattern matching is a likely future direction of my research.

4. Finally, review the model formulation. Models can be reviewed by scrutinizing the ODD protocol. In this way, the review should occur even before the model is implemented in NetLogo.

\subsubsection{Pragmatism in Individual-Based Modelling}

Individual-based modelling is characterized as describing a system by describing how the individuals within that system work (Railsback \& Grimm, 2011). The descriptive process is recursive, requiring the examination of the system's effects upon the individual while, at the same time, examining the individual's effects upon the system.

Over the years, I tracked down several lectures by Steven Railsback that were shared online. ${ }^{11}$ In particular, one lecture that was presented to a South American audience was recorded by a hand-held cellphone camera and uploaded over the course of several incomplete clips. Despite this dubious provenance, I memorialized the following Railsback quote in my notes:

\footnotetext{
11 Regrettably, all of these lectures have been lost to time. They are not online anymore and, consequently, I don't have a citation for them.
} 
You want a model that is not too simple and not too complex.

Presumably, it is only through expertise that one comes to understand when a model has the appropriate amount of complexity in order to simultaneously be descriptive of a meaningful phenomenon and also simple enough to understand. This quote speaks to the practical sensibility that underlies ecological modelling;

it is rooted in the real world, both in terms of the phenomenon and in terms of the model specification.

The pragmatic capacity to understand a model after it has been constructed can be appreciated by counter-example. A criticism of contemporary Deep Learning methods is that the models become inscrutable, such that they may solve a problem but, in doing so, they may come to defy interpretation (e.g., Doran et al., 2017). The Artificial Intelligence field has embarked on a new effort in explainable AI, which would permit interpretation for such purposes as scientific inquiry or even ethical analysis (e.g., Charisi et al., 2017).

Individual-based modelling, because it is specified by humans in the first place, does not presently suffer from this same problem. However, the potential exists for a model's complexity to outstrip the scientist's ability to understand the mechanisms that initially enabled the model to produce the desired outcomes. Individual-Based Modelers must resist this complexity. The best models, according to Railsback, will strike the appropriate balance: "not too simple and not too complex."

\subsubsection{Strong Inference}

If a scientific epistemology doesn't include a mechanism for inference, then there's virtually no way of scaffolding towards the truth. However, according to Railsback \& Grimm (2011), strong inference can be achieved with the Individual-Based Modelling approach. Therefore, it's essential for psychological science modelers to understand the tenets of Individual-Based Modelling, even though their actual work may not be ecological in nature.

Platt (1964) admonishes scientists to, “...make [intellectual] inventions, to take the next step, to proceed to the next fork, without dawdling or getting tied up in irrelevancies." The essence of the strong inferential approach is rapid hypothesis falsification, which has been adapted to the computational modelling context Railsback \& Grimm (2011, p. 245):

1. Identify alternative traits (hypotheses) for behaviour.

2. Implement the alternative traits in the ABM, testing the software carefully to get "clean results."

3. Test and contrast the alternatives by seeing how well the model reproduces the characteristic patterns, falsifying traits that cannot reproduce patterns.

4. Repeat the cycle as needed: revise the behaviour traits, look for (or generate, from experiments on the real system) additional patterns that better 


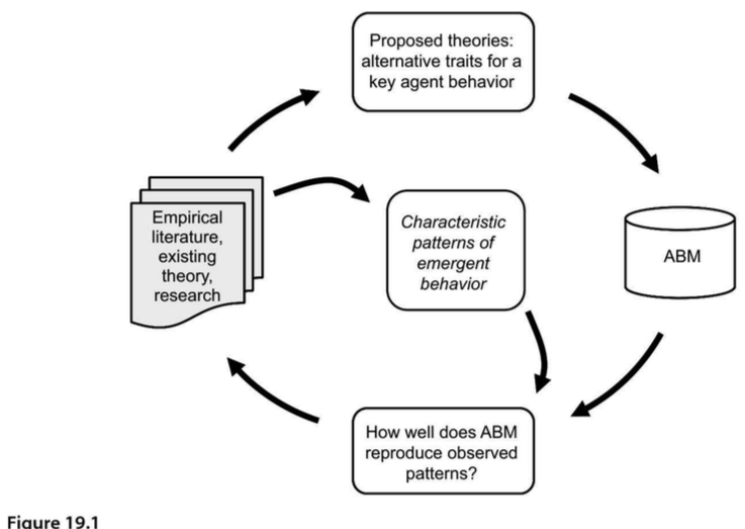

Figure 6: Railsback \& Grimm (2011, p. 245)

resolve differences among alternative traits, and repeat tests until a trait is found that adequately reproduces the characteristic patterns.

At this point, I wish to emphasize the unpredictable nature of the emergent outcomes that may be produced by simple model rules. Works such as Epstein \& Axtell (1996) are entirely non-trivial; the emergent outcomes obtained therefrom are impossible to predict a priori. It is much simpler to obtain "unwanted" outcomes from computational models than it is to actually replicate the sort of phenomena that were intended.

Inferentially, unwanted model outcomes are tantamount to falsification; when a model produces results that do not converge with empirical observation, the model is wrong - full stop. As such, the strong inference algorithm discussed in Railsback \& Grimm (2011) provides a formal framework for quickly falsifying bad hypotheses, discarding bad models and, so long as there are intellectual inventions left to investigate, finding one that works. In computational modelling, most models are both wrong and not even useful.

\subsubsection{Pattern-oriented Modelling: A Process for Falsification}

A blueprint for strong inference with computational models is called Pattern Oriented Modelling (POM) by Railsback \& Grimm (2011, p. 245). The POM procedure is reproduced in Figure 6, which and I have summarized in my own terms as follows:

1. Start with either the literature or with findings from the real world, perhaps collected in your own empirical work.

2. Identify characteristic patterns of emergent behaviour.

3. Propose theories for behaviours. A theory, for Railsback \& Grimm, is a general explanation for the pattern of behaviour, which could be expressed 
as an algorithm, a linear model, or any other mechanism. Psychology is full of theories.

4. Collect these theories together into an individual-based model, which basically amounts to expressing these theories mathematically. In practice, this is the point at which the model may be implemented with NetLogo.

5. Ask how well the model reproduces the observed patterns. The data may be analyzed like any other psychological study - or like any other natural study.

As of 2019, Null Hypothesis Significance Testing (NHST) remains the standard method in psychological science for testing how well any given model fits with observed results. However, it has become abundantly clear that this method alone is no panacea. In my experience, it does not always make sense to apply NHST in a computational modelling context - just as it does not make sense to apply NHST in every empirical context, either. Pattern Oriented Modelling, which is built upon strong inference, presents an alternative to NHST.

To Railsback and Grimm's question of, "How well does the ABM reproduce observed patterns?" we may leverage any number of criteria to produce an answer. In the current work, convergence is operationalized in terms of a model's ability to reproduce patterns of results with matching time scales, categorical levels, magnitude, and other features as appropriate. When any of these dimensions deviates, the model will be considered as falsified and it will be discarded. Only when all dimensions match will it be claimed that we have arrived at a hypothesis that will not be rejected.

\subsubsection{Psychological Patterns}

Modern Psychology is an empirical science based upon evidence and inference. In large part, psychological science proceeds according to a process of: 1) gathering insights about psychological phenomena; 2) generating models corresponding to hypotheses about those phenomena; 3) collecting new data corresponding to those models; and 4) performing inference based on the statistical properties of those models in the context of those data.

In other words, psychology is a pattern-oriented science. Therefore, the challenge of creating Agent-Based Models of psychological phenomena is a matter of incorporating psychological patterns into an ABM framework.

\subsection{The Current Work}

The current work is is an extension of the Eriksson \& Coultas model of emotional selection operating during a serial reproduction task. I formalized their model as a computational construct suitable for agent-based modelling. Those computations were then adapted to different network topologies; first, a serial reproduction task topology, as described in the background section, and then a social network topology that resembles real-world online social networks, in 


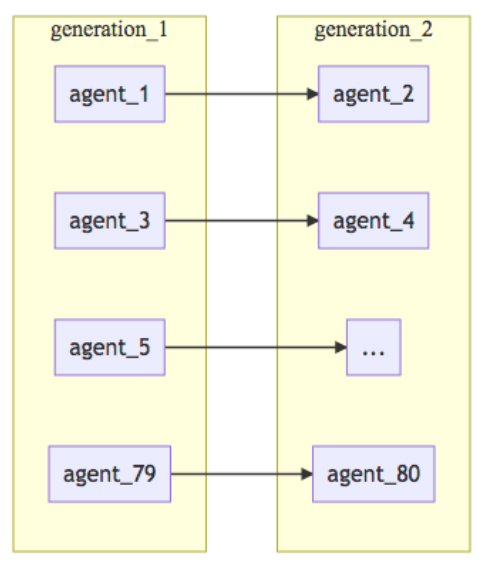

Figure 7: This basic diagram depicts the arrangement of dyads into a sequence. Stories are first provided to Generation 1 participants, then they are provided to Generation 2 participants. There is no sharing between dyads; all sharing occurs within-dyad.

which individuals have multiple connections. The goal of this work was to detect the emergence of accelerating urban legend propagation, in contrast with the original laboratory findings in which cascades terminated in fewer than two transmission generations.

I approached this task over the course of three studies.

1. First, the original dynamics of Eriksson \& Coultas (2014, Study 3) were replicated by building a computational model that matches the patterns of the original laboratory study.

2. Next, the scale of the serial reproduction study is increased by a factor of ten to explore whether sample size affects the likelihood of urban legend propagation.

3. Finally, the task is repeated using a network topology that resembles an online social network, in which individuals are permitted to have multiple connections.

\subsubsection{Study 1: Replicating Urban Legend Cascades}

Study 1 is a replication of Eriksson \& Coultas (2014, Study 3), in which the first generation participant would read urban legends and chose whether to transmit; then the second generation participant actually received those stories and repeated the task. In the ABM simulation, schematically depicted in Figure 7, there will be many little networks representing story cascades of length $=2$. Each serial reproduction task consists of two agents that are assigned as generation 1 or generation 2, which determines the directedness of the graph edge 

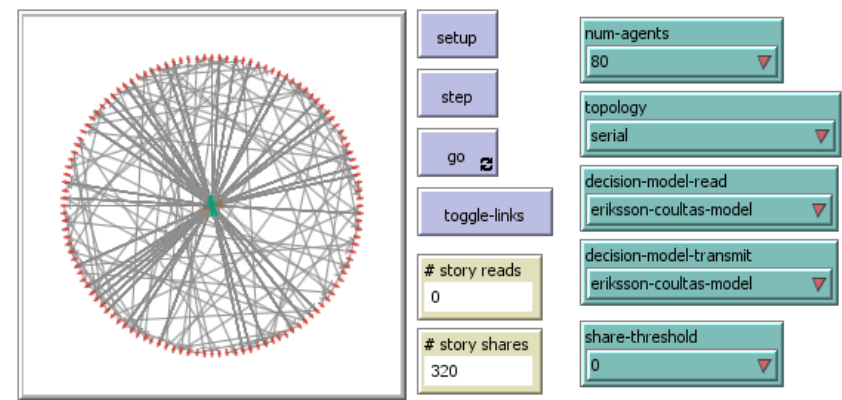

Figure 8: Study 1; NetLogo Interface. Agents are displayed in the box on the left. Buttons appearing on the right are used to interact with the simulation.

between them. Thus, the agent in generation 1 has one directed edge to the agent in generation 2 . There are 40 such dyads for a total $n$ of 80 , corresponding to the parameters of the Eriksson and Coultas study.

The study is specified using the NetLogo environment (Wilensky, 1999) and the screenshot in Figure 10 depicts the user interface developed for the task. Although agents are laid out as a circle, like the spokes of a wheel, the actual topology of the networks is exactly equivalent to the cascades specified in Figure 7. At the center of the simulation window is a special experimenter agent who is responsible for distributing stories to generation 1 agents.

The simulation begins when generation 1 agents receive stories from the experimenter agent. From the perspective of a single agent, they receive 4 stories at random from the experimenter. Then, for each story received, the agent will decide whether to read any of those stories. Of the stories they read, the agent then decides whether to share each story. Any of the stories that are transmitted are passed to the generation 2 agent.

There are two generations of agents in this simulation and only one generation acts at a time. Thus, the time duration of this simulation is 2 time units, about which more will be said shortly. Simulation data, including the number of stories retained at each generation, are logged to a CSV file, as if this study were conducted with living human participants.

\subsubsection{Study 2: Scaling Up}

Study 2 is a large-scale replication of Study 1. The intuition motivating this study addresses the question of whether the laboratory sample was too small with $n=80$ participants. Since urban legend transmission was found to be a rare event in the laboratory, then a larger sample will produce more opportunities to share, possibly resulting in a more story transmission. As far as the model is concerned, nothing changes apart from the parameter that is used to control how many cascades are initialized and how many agents are created to populate the simulation environment.

Thus, study 2 consist of 10 times as many cascades: 400 serial reproduction 

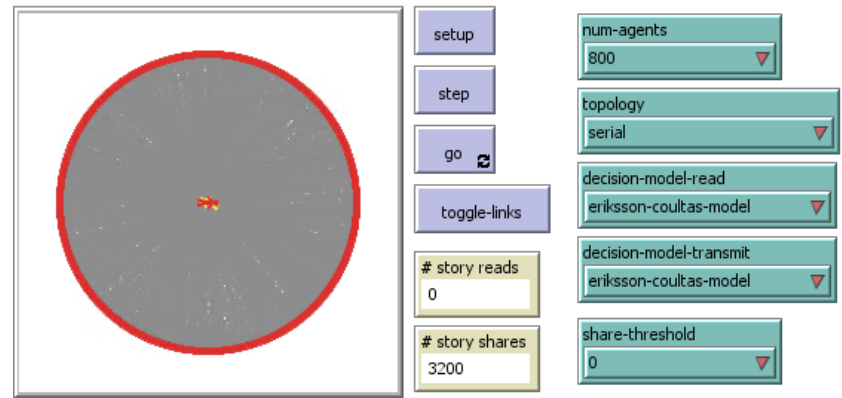

Figure 9: Study 2; NetLogo Interface. There are so many agents and connections that they all blend together. Although it is no longer possible to visualize the distinct dyads, the system is nevertheless able to keep them separate.

tasks, for a total agent sample of $n=800$. Figure 9 depicts the NetLogo user interface which is nearly identical to before, with the notable exception that now the hub-and-spoke layout appears as a solid-fill circle as a consequence of the increased density of edges. In every other way, the parameters of Study 2 are just like Study 1.

Increasing the sample size is rarely performed with human participants because it usually implies time and expense. However, computational simulations pose a different set of constraints, such that the cost and expense manifests differently. Below certain thresholds, there is virtually no marginal cost to increasing scale. Therefore, computational simulations are a cost-effective method for studying difficult-to-observe phenomena, including those that depend upon rare events, that might benefit from increased study scale.

\subsubsection{Study 3: Social Network Topology}

Study 3 is is substantial departure from the previous studies. Instead of using serial reproduction tasks, a different network topology based on a preferential attachment algorithm will be used. Preferential attachment (Barabási \& Albert, 1999) is a quick and approximate method for generating networks that have some of the same properties as online social networks. These are "rich get richer" networks in which a small number of agents have links to lots of agents; think of these as the celebrity agents. Meanwhile, the vast majority of agents are not popular; they do not have many links to other agents. Study 3 uses the same population size as Study 2: $n=800$ agents.

We will also change another detail of the original studies; these will be permitted to run a little longer - until a potential fifth generation - whereas the other studies stopped at generation 2. By permitting longer cascades, this study will afford the possibility of actually observing an increase in the number of stories that are shared over time. In the lab study, by generation 2, almost every story cascade died, so even if Eriksson \& Coultas (2014, Study 3) had specified longer cascade lengths, the effect would have been nullified because all 

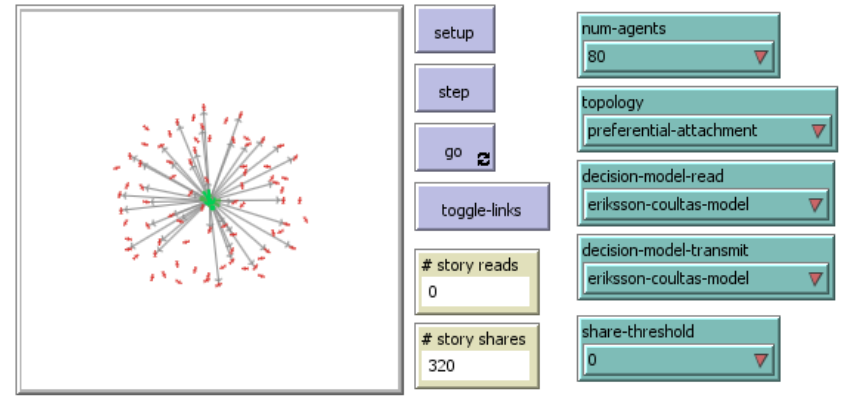

Figure 10: Study 3; NetLogo Interface with network layout. Once again, the visualization does not properly represent the topology of the networks. The elaborate network layout methods used in previous plots were not applied to this visualization. The network edges that are depicted in this visualization represent the first generation of the simulation, in which some agents have received stories from the experimenter.

low-disgust story sharing had ceased by the second generation and high-disgust stories would likely cease in the third generation. On this basis, there is no transmission advantage to running the simulation a little longer. By providing the simulation more time, it affords the opportunity to see whether the urban legend cascades die or whether they could actually become more prolific with time.

The agents act according to the rules of an S-I-S epidemic, which is a model of simple contagion that stands for Susceptible, Infected, Susceptible (e.g., Grabowski \& Kosinski, 2005). Susceptible agents, in this case, are able to become infected through exposure to urban legend stories. When agents choose to read a story, those agents become infected, recalling the parasitic model of memes that temporarily inhabit a host for as long as the story is remembered. Once agents transmits their stories - should they choose to do so - then they become susceptible again to receiving new stories in the future.

\subsection{ODD Protocol}

ODD stands for Overview, Design Concepts, and Details (Grimm et al., 2006, 2010). The ODD protocol will now be applied to the current work. In the Overview section, the model is broken down into Entities, Variables, and Process. In the Design Concepts section, the background will be summarized in order to describe why the model was formulated the way it was. Finally, the Details section describes the operation of the model.

\subsubsection{Overview}

Entities The entities or agents in this work are representative of human participants. Internally, these agents are assigned participant IDs that also determine 
the sequence in which they are activated.

Edges represent the communication channels that agents may use to communicate, thereby forming a communication network. Edges are directed so that messages only flow in one direction, which is similar to the operation of popular online social networks like Twitter. Agents may communicate only when an edge connects them.

The model also provides a representation for stories. An agent interacts with a story whenever there is reading or transmission activity. When an agent reads a story, then a readership edge is created linking the agent to the story. A story can have many readers and the number of readers can be counted by enumerating the readership edges that connect to the story. Readership edges primarily exist to track cascades as they develop.

Variables Agent behaviour is influenced by computational variables that vary between agents and conditions. These variables are used to track agent states and to create experiments within the simulation.

Has Content When an agent has one or more stories to read, that fact is tracked using a variable called has content. We can ask an agent: "do you have any stories to read?" If the answer to this question is yes, then we say of the agent that has content is true. Otherwise, the value of has content is false. As a result of formalizing the has content variable, we will be able to construct logical expressions based on knowledge about which agents have stories to read.

Has Decided Once agents have made their decisions about whether to read and share stories, this fact is tracked with a variable called has decided. Whether an agent has decided and whether they have content is relevant to an agent's eligibility to become active at any given time. ${ }^{12}$

Decision Model Agents are controlled by a special variable called decision model which is used to select between two models for decision making; either the empirically-calibrated Eriksson \& Coultas (2014, Study 2) model or an alternative called the null model, which will be described soon. Unlike previous variables, which exist to clarify the logic of the model, this variable is very consequential because it radically changes the behaviour of the agents.

Sharing Threshold Each agent has a sharing threshold that is used to transform the results of a sharing intention model, which is itself a linear regression, into a decision outcome that causes an agent to share or not. The sharing intention model is derived from Eriksson \& Coultas (2014, Study 2) and is described later in the ODD Protocol. This model of sharing is a linear function that produces a continuous value that is compared to the sharing threshold.

\footnotetext{
12 In fact, neither has decided nor has content strictly needs to exist; these values can be derived from network state. However, these two variables simplify some behaviour logic and they improve interpretability.
} 
Values above the threshold cause the agent to share a story and values below cause the agent to effectively ignore the story. As this sharing threshold could hypothetically be any number, it must be calibrated against empirical findings in order to ensure that it represents the correct threshold, a process which is described later in the ODD Protocol.

Story Variables Stories are represented as entities in this model and, consequently, they have their own variables in the same way agents do. In the laboratory study, stories were rated by participants and, consequently, data about the stories are available. Of particular relevance, participants rated how disgusting and amusing the stories were. Stories can therefore be high-disgust or low-disgust, which is captured as a single variable called disgusting that is true in the high disgust condition or false otherwise. Stories also have a categorical variable called themes, which has 4 levels corresponding to the following: cake, dog, Nepal, and pizza.

Scales Scale, here, refers to the types of dimensions that are involved in this model. Time is one scalar property of this model which proceeds in discrete increments of 1 hour, representing the duration of one experimental session. This time scale is plausible because we know the general properties of laboratory psychology research. The decisions made by participants during the Eriksson \& Coultas (2014) laboratory studies would all occur within a single experiment block.

Similarly, a single time tick is the amount of compute resource allocated to performing the decisions that occur within a single experiment block. Thus, we establish a correspondence between one hour, one experiment session, and one time tick within the simulation. When replicating a 2-link serial reproduction task, think of it as two separate, one-hour sessions that may be summed to 2 hours.

Process overview and scheduling In agent-based modelling, time itself may be conceived of according to various architectures. All simulations proceed in a certain order that is determined by a schedule. This simulation activates agents in the same order during every time tick, and that order is determined by the agents participant ID; lowest, first.

A consequence of this schedule, in which all agents act at every time tick, is that all generation 2 agents are updated during time tick 1 - even though it is not actually their turn to act. Likewise, all generation 1 agents are updated during tick 2. The variable called has decided therefore prevents agents from acting when it's not their turn, permitting agents to logically skip a turn when appropriate. Once an agent completes its actions, then the next agent begins according to participant ID.

Another consequence of this schedule is that agents do not act in parallel, which is a departure from all our assumptions about how our lived (nonsimulated) experience of reality operates. Only one agent is computed at a 
time but, importantly, agents do not actually "notice" when they are paused. That is, from an agent's perspective, it is as if all agents exist within a parallel, simultaneous world. However, from our perspective as researchers who are situated outside the simulation, we have the knowledge that agents do not act simultaneously - although agents are entirely unaware of this quirk.

\subsubsection{Design Concepts}

Interaction Interaction describes the ways Entities of various kinds (i.e. agents, stories) can influence one another in the simulation. In this model, there are several possible kinds of interaction, depending on the types of entities that are interacting. The flowchart in Figure 4, which describes the methods used in Eriksson \& Coultas (2014, Study 3), also happens to describe all of the major interactions among entity types.

Communication Communication is one type of interaction that occurs between two agent entities. In this model, communication occurs by collecting urban legend stories into a packet that is transmitted the next agent in the cascade.

Reading and Sharing Other forms of interaction occur between Agents and Stories. When an Agent chooses whether to read a story, that is modeled as an interaction between a Story entity and an Agent entity. Agents also interact with stories when they decide whether to share them.

In the current research, each of these forms of interaction has network implications. Communication may only occur along existing network routes. Meanwhile, the read and transmit interactions cause new network links to be created that encode the consequences of the interaction.

Stochasticity There are several random processes in the computational model, many of which are modeled as Gaussian noise. In the case of the linear regression models that were used to define the behaviour of the model entities, responses were centered and standardized during the model fitting process which is calculated under the assumption of normality. So, I added Gaussian noise like a residual term to the fitted model estimates that were reported by Eriksson and Coultas to allow different entities to behave slightly differently from one another.

Emotional selection during the reading phase results in a non-normal distribution of choose-to-receive decisions. I model these choose-to-receive decisions as events over time, which I derive from the Poisson distribution. The mean receive rates that are reported in Eriksson \& Coultas (2014, Study 3) determine the parameters of this Poisson distributions. As with Gaussian processes, this linear model may be operated in reverse - "made stochastic" - by sampling randomly from the Poisson distribution to add to the function where the error term ought to be. 
A different kind of random process is used to generate preferential attachment networks in Study 3, which is intended to simulate social network dynamics. The preferential attachment algorithm links new agents to another agent in proportion to the number of existing links an agent has. In this way, agents with many links have a higher likelihood of receiving even more links over time. This preferential attachment algorithm produces similar but slightly different topologies each time it is performed.

Emergence We expect several non-obvious patterns to emerge from this. The number of stories retained per generation is the primary emergent outcome that will be used to compare laboratory results with simulation results. The number of stories retained can be computed on a per-cascade, per-individual, and perstory basis. We expect to see that high-disgust stories will be retained at a higher rate than low-disgust stories.

Another pattern we expect to emerge is Story extinction for serial reproduction topologies, in order to replicate Eriksson and Coultas. Based on their laboratory results, low-disgust story cascades should completely "die off" within 2 generations. In contrast to story extinction for serial reproduction cascades, we expect social network topologies to manifest ever-increasing shares over time.

Observation The simulation must permit the observation of the total number of Stories shared at each generation. In order to collect data about the computational model, the simulation environment must be monitored by a data logging process. For this purpose, NetLogo provides tools for observing emergent properties of the model while it is running, which can be programmed to log the number of shares per cascade, per generation, and so on. These observations may then be exported to a CSV file. NetLogo also enables direct interaction with the simulation using Graphical User Interface controls and outputs.

\subsubsection{Details}

Initialization The process of initializing the simulation starts with the creation of all the agents in the environment, then connecting those agents with a network. Study 1 and 2 produces a serial reproduction topology to connect the agents. In study 3 , a preferential attachment network connects the agents according to the stochastic process previously described. As the last step of initialization, the Experimenter agent is connected to generation 1 agents who then transmits stories to those agents in order to prepare the simulation before time starts.

Step Model Each time tick proceeds according to an algorithm called step, depicted in Figure 11, which is responsible for describing what happens during a time tick. Time itself moves forward as a progression of these steps.

At the first branch in the flowchart, when an agent has no stories during a step, then the agent does nothing. Otherwise, once some stories have been 


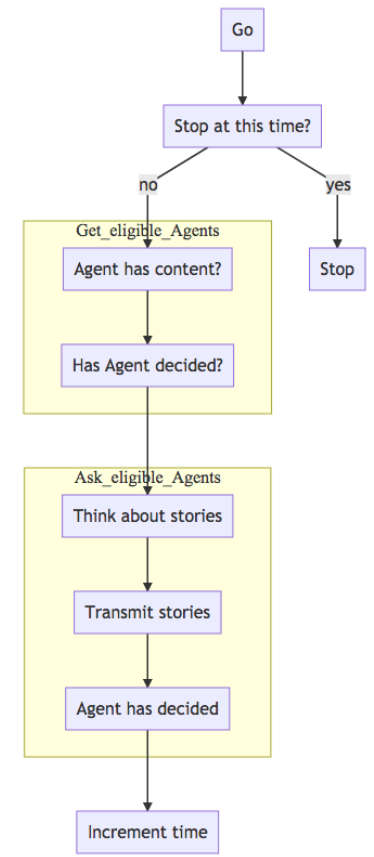

Figure 11: This is a flowchart depiction of ABM "step" submodel. These labels represent the simulation sequence in a very rough, conceptual manner, as viewed from the perspective of the simulation itself. The behaviour of individual agents, which has been described elsewhere in the ODD protocol, can be thought of as being situated within the steps of this flowchart. 
transmitted to the agent, the agent performs the choose-to-receive behaviour and, for any stories they read, performs the choose-to-transmit behaviour.

choose-to-receive Model The receive phase of the communication model is represented as an algorithm called choose to receive. Eriksson \& Coultas used the name "choose to receive" to describe what their participants do when they decide whether to read a given story. The computational model therefore has a corresponding representation with the same name.

According to Eriksson \& Coultas (2014, Study 3), there is a significant difference in story retention during the choose-to-receive phase, depending upon whether the story is high-disgust. In case the story is high-disgust, then the mean number of stories retained is significantly higher - a mean of 2.4 stories versus a mean of 1.2 stories for low-disgust.

When the story is disgusting, the choose-to-receive algorithm is more likely to produce a result that causes the agent to read the story. This is modeled as a Poisson process that determines the likelihood that a specific story will be retained, depending on disgust level. In proportion to that likelihood, chooseto-receive randomly reports either "yes: read the story" or "no: skip it."

choose-to-transmit Model The choose-to-transmit algorithm derives from Eriksson \& Coultas (2014, Study 2). Agents will share based on two quantities: first, what is the predicted share score for the agent and story; and second, what is the threshold above which agents will share. The prediction function is derived from the results of the regression analysis conducted by Eriksson \& Coultas. I think of this prediction function as a regression in reverse. Instead of fitting a model to a fixed set of data, the model is used to generate a brand new set of outcomes. When the score produced by this function is above the share threshold, the agent has effectively chosen to transmit a given story.

$$
\begin{array}{r}
\text { share_score }=-0.86+(0.79 * \text { disgust })+(0.08 * \text { social })+(0.22 * \text { amusing })+ \\
(0.35 * \text { interesting })+(0.25 * \text { plausible })+(0.18 * \text { surprising })
\end{array}
$$

To compute a linear regression in reverse, we sample from the Gaussian distribution for each parameter in Equation 1. As discussed in the stochasticity section, a critical assumption of the analysis is that values are zero-centered and standardized. The products and sums are computed to obtain the share score.

Null Model of Behaviour Until this point in the ODD Protocol, we tied our model specification to the empirical context of the Eriksson \& Coultas (2014, Study 3). Now, we consider an alternative theory so we can potentially falsify our computational model; if it is no different from the null model, we ought to reject it. This requires a rhetorical straw man of sorts, analogous to the null hypothesis that is familiar to frequentist significance testing.

What happens when agents behave in a purely random manner? To implement this, we can model the receive and transmit decisions as the result of 
a uniform random process. $50 \%$ of the time, agents receive the stories. $50 \%$ of the time, agents transmit the stories. I call this random decision model the null model - and, throughout the results, I refer back to various null models of behaviour for comparisons.

I specifically think the null model does not describe the way people behave in real life. I expect that when agents behave according to the null model, it will not resemble the Eriksson \& Coultas (2014, Study 3) results. However, if it turns out that the null model produces results that are as good as the computational model, then we have no choice but to reject the theory underlying the computational models and try again.

\subsection{Simulations}

\subsubsection{NetLogo}

The NetLogo agent-based modelling environment was used for this work. The simulation works with NetLogo version $6.0 .4 .^{13}$

\subsubsection{BehaviorSpace}

The NetLogo BehaviorSpace ${ }^{14}$ tool is used to iterate across a specified parameter space, repeatedly running models with varying parameters. The results from BehaviorSpace may then be logged to a CSV file. BehaviorSpace was used extensively in the present research to create structured batches of simulations that generated the raw data. Once a CSV file was obtained from BehaviorSpace, it could be imported into a statistical package for statistical analysis.

\section{Results}

\subsection{Study 1: Replication}

The first objective of this research was to replicate the pattern of results observed in Eriksson \& Coultas (2014, Study 3). These patterns can be characterized as follows:

1. High disgust stories propagate at a higher rate than low disgust.

2. Fewer stories are received and transmitted as time proceeds.

3. At each time point, fewer stories are transmitted than received.

4. By the end of two time points, virtually no low disgust stories are transmitted.

13 The NetLogo language has changed slowly over the course of the years. I expect that this code will only work with this specific version of NetLogo. My recommendation for reproducibility is to archive a complete copy of NetLogo along with the model specification.

14 The Canadian English spelling, BehaviourSpace, would produce no results. 


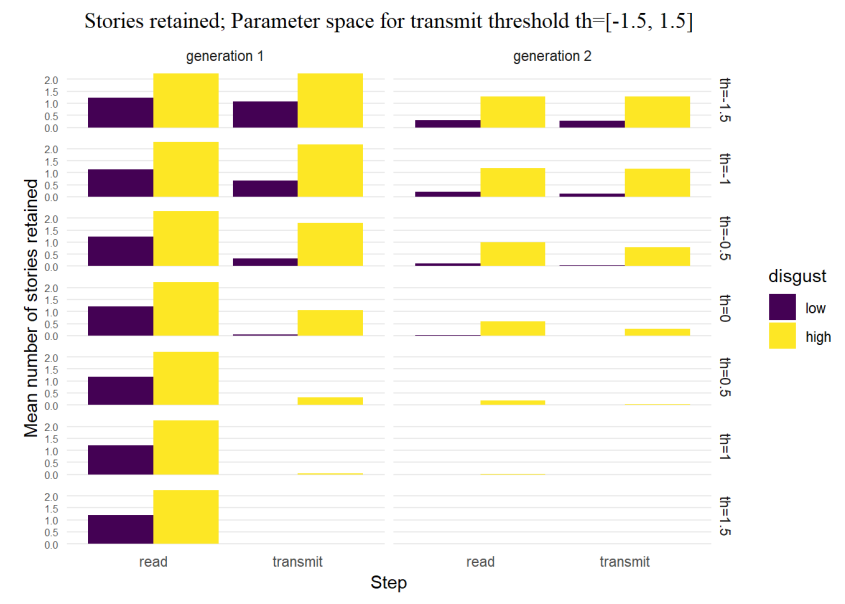

Figure 12: This plot depicts the results of the search for a share_threshold parameter. The simulation was executed with different threshold values, which are abbreviated as th and can be seen along the right-most edge of the plot. Thus, the different parameter results are actually stacked on top of one another.

\subsubsection{Calibrate share_threshold parameter}

Before producing the results, there was one model parameter that constituted a degree of freedom in the simulation model. The share threshold, which was created in order to transform a regression model into a decision-making function, was not specified by the original research. As this parameter has no direct empirical basis in Eriksson \& Coultas (2014, Study 3), the parameter must be calibrated. ${ }^{15}$

A sensitivity analysis was performed upon the share threshold parameter (Thiele et al., 2014). The parameter space between -1.5 and +1.5 was searched in increments of 0.5 . During sensitivity analysis, $k=30$ simulation runs were performed with each threshold value. The plot in Figure 12 visualizes the parameter space for share threshold along with corresponding results.

Mathematically, the share threshold may be interpreted in terms of its computational consequences. When the share threshold is 0 , then a transmit intention score of 0 and above will trigger transmission. When share threshold is below zero, agents share at an increased rate - and the farther below 0 the threshold is, the more transmission there is. When share threshold is above zero, there is less transmission.

When the threshold is 0 , not enough stories are left by the end of the second

\footnotetext{
15 Any such degree of freedom yields obvious criticisms for agent-based models. As such, great care was taken to eliminate any other degrees of freedom during the construction of the ODD Protocol. Nevertheless, this one degree of freedom remains. Luckily, this problem is not unique in simulation research and the method of Sensitivity Analysis was developed to solve it.
} 


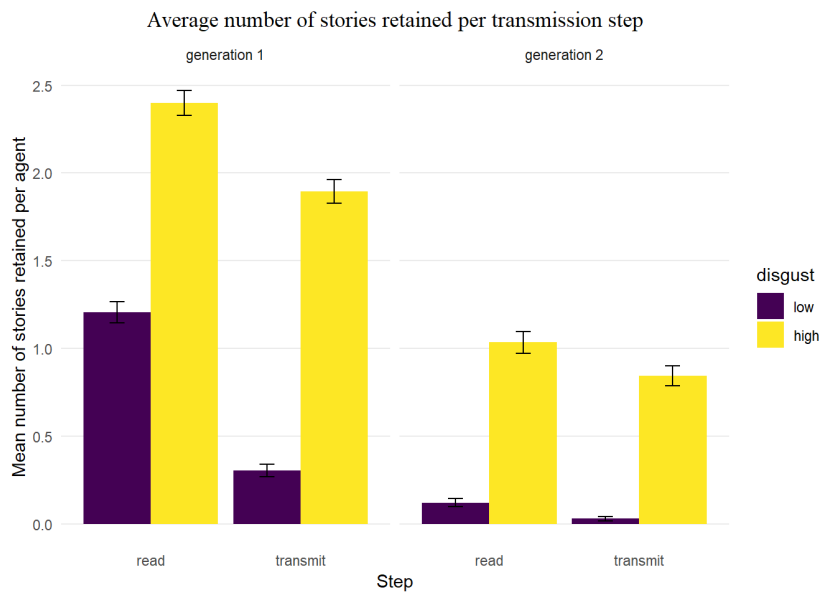

Figure 13: The results of Study 1 correspond closely to the empirical results obtained by Eriksson \& Coultas.

generation. When the threshold is -1.5 , there are too many low-disgust stories. On the basis of these parameter sensitivity results, a share threshold value of 0.5 was selected for all simulations.

\subsubsection{Simulation Results}

Figure 13 depicts the results obtained from $k=30$ simulations performed with NetLogo BehaviorSpace. As with Eriksson \& Coultas (2014, Study 3), there are 2 generations of agents in which there is a receive and a transmit step. These results are directly comparable to Figure 2.

Simulation results converge with the absolute magnitudes that were reported in Eriksson \& Coultas (2014, Study 3). When focusing on the generation 1 receive step, on average agents receive a little more than 2 high-disgust stories and a little more than 1 low-disgust story. During the generation 1 transmit step, nearly 2 high-disgust stories are transmitted, on average, whereas just 0.25 low-disgust stories are transmitted.

Overall, the simulation produces a similar magnitude of reading and transmission events over time. Directly evaluating the patterns yields the following:

1. The simulation produces different outcomes for high versus low disgust stories. High disgust stories are transmitted at a higher rate.

2. Stories with different disgust levels are retained at an appropriate rate over time.

3. Within a single generation, the number of stories transmitted is lower than the number that were received. 


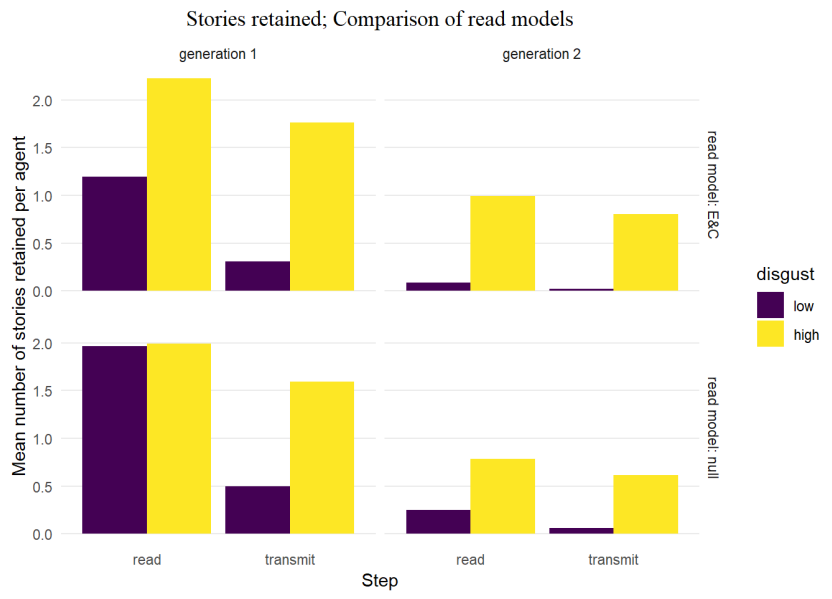

Figure 14: When the behaviour for the choose-to-receive submodel is determined by a random source, instead of the empirically calibrated model, the simulation results no longer match the pattern of the empirical results. The plots are stacked: read model: $E \mathcal{E} C$ refers to the Eriksson \& Coultas model for choose-to-receive. Meanwhile, read model: null refers to the random model.

4. By the end of two generations, low disgust stories are almost entirely extinguished - yet some high disgust stories remain.

Altogether, the overall pattern of stories retained over time resembles the original Eriksson \& Coultas (2014, Study 3) results.

A Brief Note About Error Bars The error bars in Figure 13 occur because each simulation run produces a slightly different result. The size of the error bar is proportional to the number of simulations that were run. This is very different from the error bars that occurred in Eriksson \& Coultas (2014, Study 3 ), which result from between-subjects variability. This topic will be revisited during the discussion.

\subsubsection{Compare choose-to-receive null model}

The choose-to-receive decision model was investigated by comparing it to a null model of behaviour. ${ }^{16}$ An unbiased choose-to-receive model was created that randomized decisions at a $50 \%$ rate, rather than determining decisions according to the empirical regression model. This is referred to as a null model because it is analogous to the no-difference condition of null hypothesis significance testing. In case the null model appears identical to the choose-to-receive model, we would

\footnotetext{
${ }^{16}$ We cannot simply "turn off" the receiving behaviour. When disabling it entirely, the net effect would result in none of the agents ever doing anything because everything depends on what the agents read.
} 


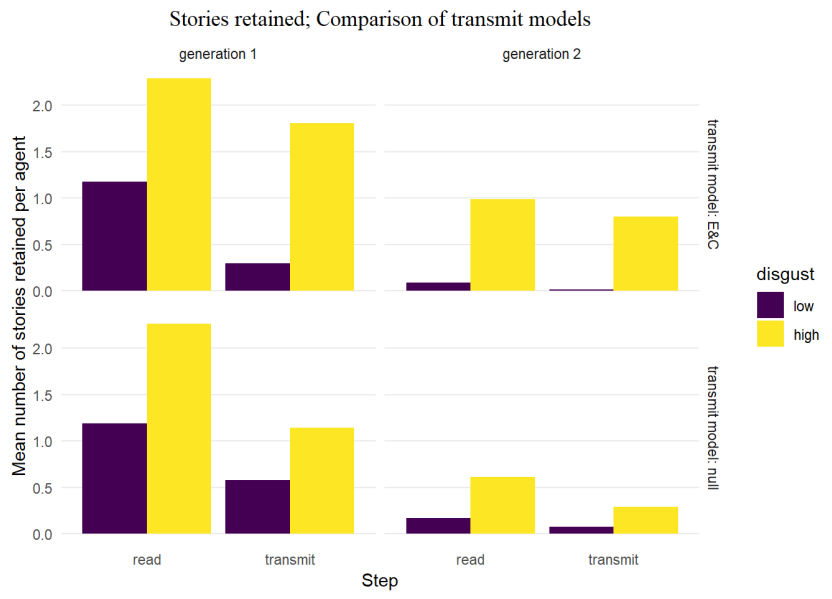

Figure 15: When the behaviour for the choose-to-transmit submodel is determined by a random source, instead of the empirically calibrated model, the simulation results no longer match the pattern of the empirical results. The plots are stacked: transmit model: $E \mathcal{B} C$ refers to the Eriksson \& Coultas model for choose-to-transmit. Meanwhile, transmit model: null refers to the random model.

conclude the choose-to-receive model adds no information. When no difference is detected, the choose-to-receive model is regarded as falsified.

Figure 14 depicts the null model and the choose-to-receive model. The choose-to-receive model results are actually a full replication of the previous results, not merely a duplication of the plot that was already presented. ${ }^{17}$ The plot along the bottom was created by running the simulations with the null model for reading behaviour.

As before, there are two generations, each containing a receive and a transmit step. The problem with the null model is evident in the first generation receive step. Almost exactly 2 stories are received by agents, regardless of whether the stories are high or low disgust. The null model result deviates from those that were expected from the Eriksson \& Coultas (2014, Study 3) model. The consequences of the null model behaviour propagate throughout subsequent steps and generations, violating expectations at each step.

Because the empirically-calibrated model differs from the null model we do not reject our initial model at this point.

\subsubsection{Compare choose-to-transmit null model}

The choose-to-transmit model was also examined by comparing to a null model. Figure 15 is laid out like the previous slide: the top depicts the empirically-

\footnotetext{
17 Since I am able to run a batch of simulations in a matter of minutes, I just re-ran the simulation instead of copying the data already produced.
} 


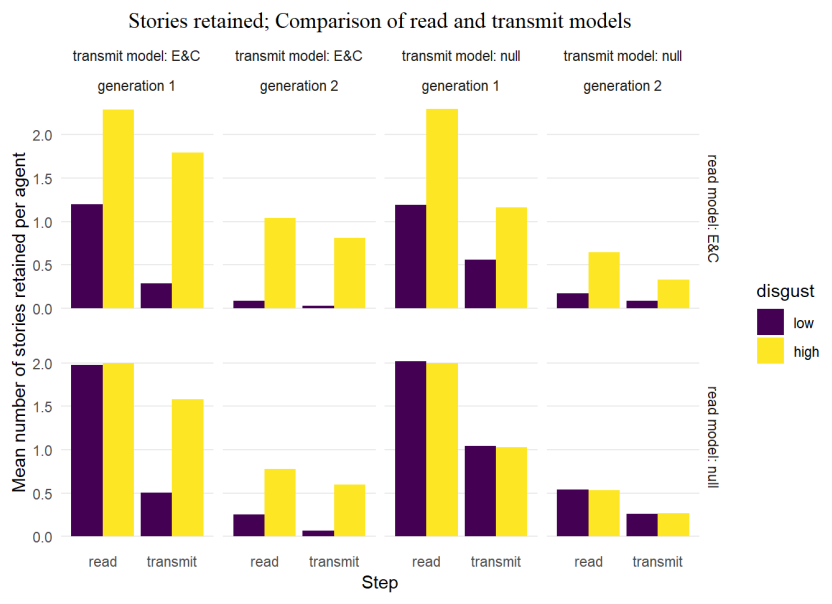

Figure 16: Plots are both stacked on top of one another and aligned side-byside. The lower-right quadrant depicts the case when null models are used for both receive and transmit. Meanwhile, the upper-left quadrant depicts the case when both receive and transmit are based on the Eriksson \& Coultas model. By comparing the upper-left to the lower-right, it can be seen that the null models fail to capture any of the patterns that were sought after.

calibrated model and the bottom depicts the null model. In the null model, high-disgust stories are not retained at a high enough rate and low-disgust stories are retained too often, as compared to the empirically-calibrated model.

By the end of the second generation, the number of retained stories drops off geometrically; at each generation, half as many stories are retained. This observation is consistent with the expected value of the null model, which will randomly transmit $50 \%$ of the stories. From this, we can infer that the null model for choose-to-transmit does not capture the transmit dynamics that were observed in Eriksson \& Coultas (2014, Study 3).

As before, we do not discard the choose-to-transmit model in favor of the null model.

\subsubsection{Compare both null models to empirical model}

Figure 16 depicts the number of stories retained when the null model is used for both receive and transmit behaviours. Only the plot in the bottom-right quadrant is new; the other three plots are replications of the results from the previous slides.

The null-transmit-null-receive model appears to decrease geometrically by a factor of 0.5 , corresponding precisely to a 50/50 coin flip. At the beginning of generation 1,4 stories becomes exactly 2 stories in the read step, which becomes exactly 1 story in the transmit step. This pattern of division, which continues through generation 2 , clearly does not resemble the original laboratory results. 


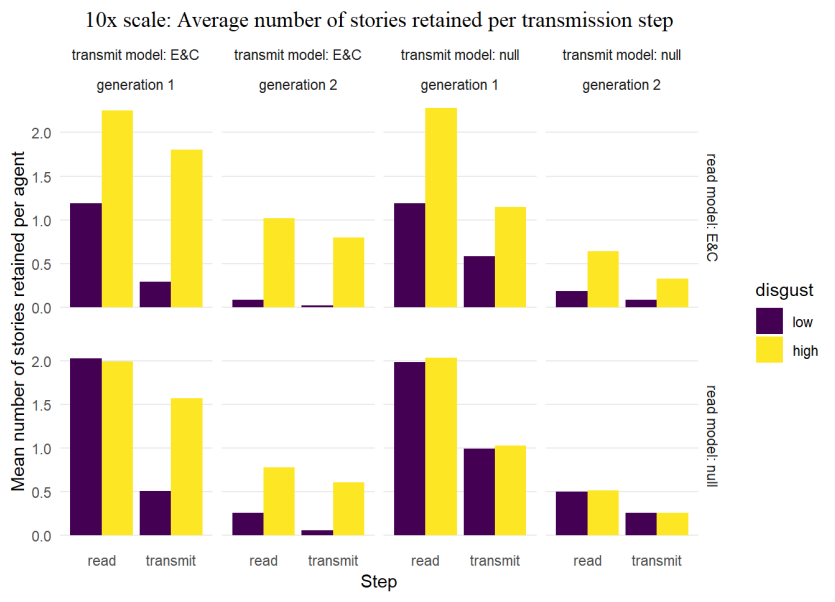

Figure 17: The Study 2 results are laid out in quadrants, as in Figure 16, and can be interpreted in the same way. The general results are preserved: the null results capture none of the phenomenon. Although the scale of this simulation has been increased by a factor of 10 , there is no change to the overall pattern of results.

By looking at all 4 quadrants in Figure 16 simultaneously, it is possible to mentally "partial out" the relative contributions from the empirical models for receiving and transmission. The effect observed in Eriksson \& Coultas (2014, Study 3) can be thought of as either being greater than or less than the corresponding null models.

\subsection{Study 2: Receive-transmit pattern at $10 \mathrm{x}$ scale}

Urban legend transmission in Eriksson \& Coultas (2014, Study 3) was a relatively unlikely event. Perhaps the reason the results don't resemble the widespread urban legend sharing observed in the real world is that sharing is so unlikely that it simply could not be seen with $n=80$ participants. Therefore, increasing the number of sharing opportunities could increase the likelihood of observing a cascade that extends beyond 2 generations.

In Study 2, a computational model with $n=800$ agents was examined. This size increase is a factor of 10 beyond the Study 1 simulations. The serial reproduction task length remained fixed at $l=2$, yielding 400 transmission tasks.

The results are presented in Figure 17. As before, null models were simulated for both receive and transmit behaviours. The top-left quadrant depicts the full empirically-calibrated simulation and the bottom-right quadrant depicts the full null model.

Despite increasing the simulation scale by a factor of 10 , the results appear virtually identical to before. None of the base rates for any particular behaviours 


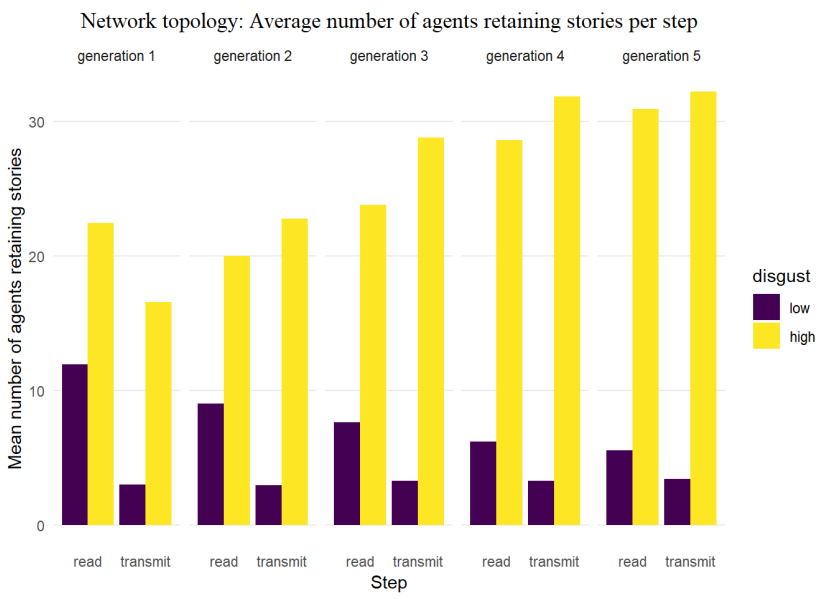

Figure 18: Study 3 Results. The empirical Eriksson \& Coultas model - both receive and transmit - was used to produce this plot. The difference is that now, a preferential attachment network topology was used. Along the $x$-axis, there are now 5 generations instead of 2 , because the simulation was permitted to run a bit longer. The important thing to take note of is the increase in high-disgust stories that were shared by generation 5 . If this pattern were continued into the future, it is now plausible that some of those stories could produce widespread cascades.

has changed. The net effect of the increase in scale is likely to just increase the precision of the estimates produced by the simulation. Although complex systems are capable of producing unexpected results under certain conditions, it does not appear that this particular system is affected by the sample size.

\subsection{Study 3: Preferential Attachment}

Until this point, these studies have been replications of a serial reproduction task and the very simple network topology implied thereby. What happens when a more sophisticated network, simulating more realistic social phenomena, is used instead? As explained in the methods, the new network is built with a preferential attachment algorithm. Consistent with Study $2, n=800$ agents.

\subsubsection{Reporting Total Stories versus Average Stories}

Figure 18 depicts the total number of stories retained over time. In studies 1 and 2, averages were reported because that's how Eriksson \& Coultas (2014, Study 3) reported it. However, in Study 3, a different metric for reporting is used so a few words need to be said about it.

With the new network topology in Study 3, agents may have many incoming and outgoing connections. Agents are activated at each generation depending 


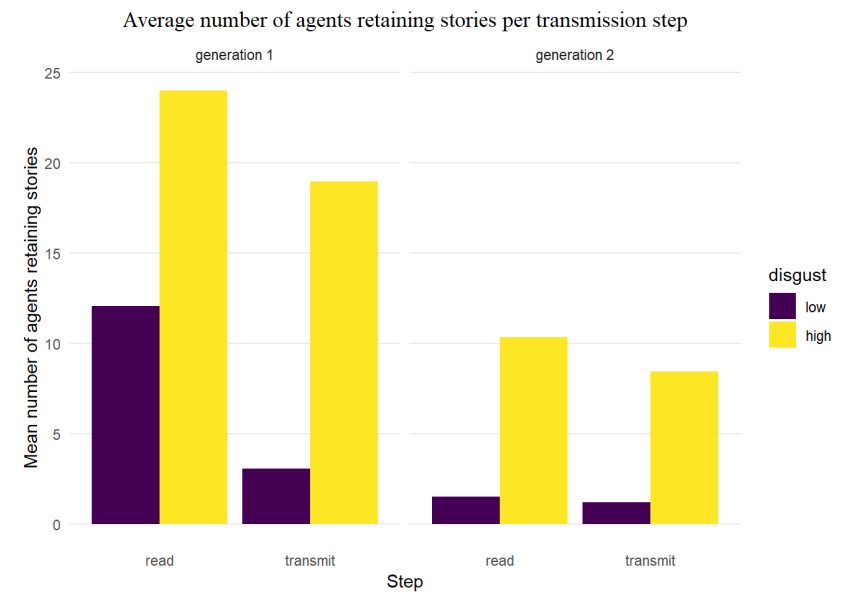

Figure 19: The results from Study 2 are plotted again - this time, as total stories per generation instead of average stories per generation. Despite this change in reporting, there is no substantial difference to the results; the same pattern is observable.

upon which agents transmit urban legend stories to them. Therefore, generations no longer consist of a fixed size. As a consequence of these differences, there's not really a denominator to use for the calculation of an average on a per-generation basis. Instead, the non-cumulative total stories retained at each generation were plotted in Figure 18.

In order to retain the ability to make comparisons to the previous studies, the results from Study 2 results have been re-plotted in Figure 19 using the total stories retained. As can be seen, the same patterns are observable; there is no substantial difference whether Study 2 results are plotted by total or by average. Therefore, the ability to interpret Study 3 results should be preserved.

\subsubsection{Simulation Results}

When examining Figure 18, generation 1 looks identical to before. However, in generation 2 and beyond, something different is happening. Instead of the number of stories gradually decreasing, the opposite happens: more high-disgust stories are retained in generation 2 than in generation 1 - and this trend continues. The low-disgust stories, as before, are retained less by each successive generation.

This result matches our original intuition about urban legends. In contrast to the effect observed in Eriksson \& Coultas (2014, Study 3), the very same model produces "viral" sharing when the network resembles a human social network. Furthermore, this effect only holds for high-disgust stories, not for their low-disgust counterparts. 


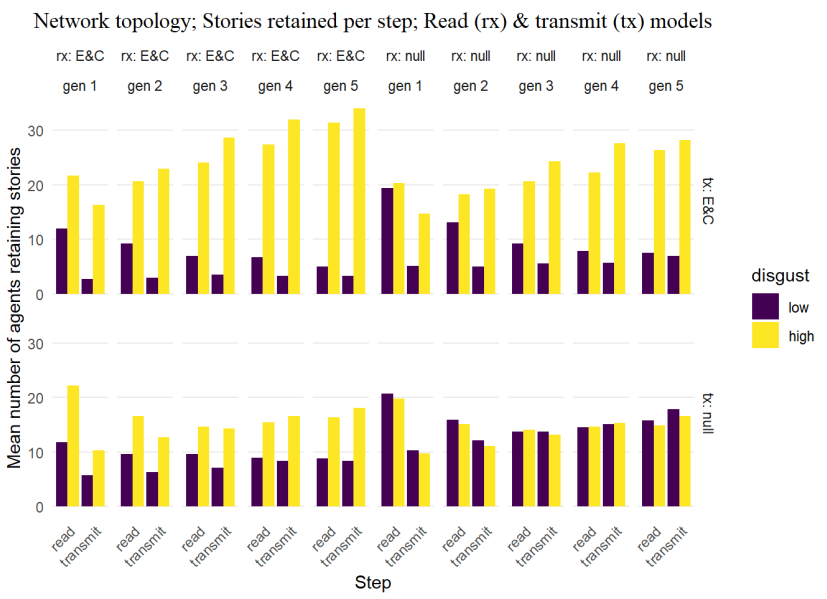

Figure 20: To permit comparison with the null models, the Study 3 results are plotted in quadrants again. The upper-left quadrant depicts the empirical models and the lower-right quadrant depicts the null models. In this plot, the term $r x$ was used to abbreviate "receive" and the term $t x$ was used to abbreviate "transmit." Although the full null model does exhibit an increase in stories over time, it can be seen that the expected patterns are not present.

\subsubsection{Null model with Preferential Attachment topology}

Null models of receiving and transmission were examined with the preferential attachment network. These results are plotted in Figure 20. The lower-right quadrant, in which both null models used, exhibits a gradual positive trend over time, suggesting that the network topology alone contributes strongly to the effect.

Critically, the null model does not exhibit any preference for one type of story over the other, which violates key patterns. On that basis, we do not reject the empirically-calibrated model.

\subsection{Summary}

Study 1 replicated the results of Eriksson \& Coultas (2014, Study 3). A sensitivity analysis was performed for the share threshold parameter in order to calibrate it for all subsequent simulations. Null models for receive behaviour and transmit behaviour were examined in order to test whether the Eriksson \& Coultas (2014, Study 3) models should be discarded. The null models did not reproduce any of the patterns whereas the empirically-calibrated models did. Thus, the empirical models were not discarded.

In Study 2, the size of the simulation was increased by a factor of 10 in order to to look for any sort of scaling properties. It was observed that the number of serial reproduction tasks did not change the outcomes for urban legend sharing. 
In Study 3, the network topology was constructed using a preferential attachment network in order to resemble a human social network. With this new network, an ecologically-plausible outcome was obtained. Unlike in the lab, the high-disgust stories increase over time, just as can be observed in the real world.

\section{Discussion}

\subsection{The Lab and The Simulation}

We began with urban legend studies that were highly controlled. Although those studies lacked ecological validity and the results didn't resemble real life, they had excellent internal validity. A computational model of urban legend transmission was constructed on the basis of those studies. The simulations exhibited the patterns that were expected, reproducing both the laboratory results and results that would be expected in the "real world." Using a realistic network, high-disgust stories are shared at an increasing rate. Low-disgust stories, which are retained at ever-diminishing rates, would likely be forgotten entirely if the simulation were run longer.

\subsection{Scaling a serial reproduction task}

Increasing the scale of the serial reproduction task did not change the rate at which stories were retained. The pattern of results with 800 agents was the same as with 80 agents. This is actually interesting on its own because most other network topologies are parameterized in some way by scale. Serial cascades would appear to be unique in their stability under scaling conditions. On this basis, perhaps the serial reproduction topology is particularly well-suited to experimental control.

\subsection{Explanations for Emergent Network Behaviour}

A key pattern exhibited in Study 3 by the preferential attachment network is that high disgust stories are retained more, not less, at generation two compared to generation one. Why does this increasing-transmission behaviour emerge with the preferential attachment network?

Revisiting an earlier intuition, urban legend transmission is a rare activity - but increasing the number of opportunities to share counteracts the model's general bias against sharing. The preferential attachment topology permits agents to be connected to more than one other agent. By changing the indegree and out-degree, this produces far more transmission opportunities.

There are epidemiological implications to the preferential attachment network. Whereas the serial reproduction task permitted agents to act just once, now agents could potentially act more than once due to the social-networklike connections they have. In epidemic ABM terms, the preferential attachment network acts as an S-I-S simulation - or susceptible, infected, susceptible. Agents that are available to receive urban legend stories are called susceptible, 
so the return to susceptibility in an S-I-S model implies that agents can receive and transmit stories multiple times.

The network topology and epidemic model therefore radically changes the range of possible agent behaviours, going far beyond the serial reproduction task. In this manner, there is a larger difference between Study 2 and Study 3 than might be immediately apparent. That algorithmic change, along with its consequences for agents, is the difference that causes Study 3 to have ecological validity when Study 2 does not.

\subsection{Messages and Topologies}

In their article, Eriksson and Coultas remarked that, "a link between emotional selection and social network structure might act as a factor that magnifies the cultural differences between communities, an idea left for future research."

I would like to rephrase their comment as follows: what about those messages that produce changes in networks? Using the nomenclature of Twitter or Facebook, what happens when we unfriend people? For example, what happens when a political conversation turns nasty and, as a result of that conversation, you don't want to be friends with that person anymore? Social networks are dynamic - and a message can cause us to add people to our network or remove them.

It is surprisingly difficult to obtain data from Twitter and - especially - Facebook that would be suitable for examining the unfriending question. However, I can do some satisfying work with computational modelling. I presented some simulation findings on the topic of unfriending at the Political Networks 2018 conference. This is a future direction that I am still interested to pursue.

\subsection{Error Bars and Parallel Simulations}

The error bars produced by the simulations are different from the error bars reported in the laboratory studies. In both cases, the error bars are fundamentally related to the uncertainty surrounding estimation. In the case of human subjects research, individual observations correspond to the measurements from one individual participant and are aggregated as a between-subjects measure. In the case of simulation studies, the results are aggregated at the scale of an entire simulation as a between-simulations measure.

The error bars reported by Eriksson and Coultas represent the uncertainty surrounding the estimates of population parameters. However, there is fundamentally just one population and we presume there is a single, unified reality that all participants belong to. Those error bars, as determined by the sample obtained, are related to the confidence in the estimation of the true mean.

Despite starting with the same parameters each time, every simulation run produces results that are a little bit different due to stochasticity. When summarizing across simulation results, we obtain an estimate of an emergent parameter

produced by a complex system - and that estimate has error. In effect, we are 
sampling from parallel simulations. ${ }^{18} \quad 192021$

\subsection{Statistical Inference}

During the analysis of the simulation results, it became apparent that the tightness of error bars could be controlled by changing how many times the simulations were run. In order to obtain a better estimate of an emergent simulation parameter, the simulation could be run more times.

This is reminiscent of the way frequentist statistics are sensitive to sample size; similarly, in order to obtain a better estimate, just obtain more observations. Gelman \& Shalizi (2013) extended this intuition a step farther by claiming that $\mathrm{p}$-value can be viewed as a crude measure of sample size, which is to say that if a specific p-value is desired, sample size can always be increased.

In the context of the current computational modelling work, I have come to wonder whether the following statistical inference process is conceivable: First, let us contrast two theories - say, the Eriksson and Coultas choose-to-receive theory against a null model of choose-to-receive. Next, run both simulations repeatedly, measuring our key emergent property of interest. Finally, determine the likelihood that the values obtained from each theory are drawn from separate distributions.

The intuition underlying this process is like Gelman in reverse: perhaps the number of simulation runs is a crude measure of p-value. When more simula-

\footnotetext{
${ }^{18}$ By implication, these are parallel simulated realities implied by the computational simulations. As a stochastic system, each simulation does correspond to slightly different starting parameters, although the parameter that varies - a random seed - exists as part of the simulation environment, not as part of the model itself. This stochastic seed value, alone, is responsible for the chaotic unpredictability that manifests as the distribution that produces the error bars.

19 The error bars therefore represent the uncertainty obtained from measuring parameters across parallel simulations. In this framework, the only parameter that varies is simply an index (i.e. the random seed) corresponding to which parallel simulation is being observed. So long as that index is recorded, it is then possible to re-run the simulation to obtain precisely the same results, which amounts to the repeatability of history.

${ }^{20}$ When this thought experiment is applied to the universe within which we find ourselves, a shocking correspondence to theoretical physics is obtained. Just as parallel simulations may be dimensionalized as an index according to the random seed that implicitly perturbed each run to produce slightly different results, a similar index is conceived of in M-theory, which gives rise the set of all possible universes (Banks et al., 1997). One advantage to M-theory is that it has already mapped out the possible dimensions that may be parameterized, although those dimensions are physical in nature and the underlying model produces physical constants like gravity instead of urban legend propagation.

${ }^{21}$ Ultimately, this thought experiment leaves me wondering about the underlying stochasticity of the observed universe. What is randomness, after all? Where, in the natural world, does the normal distribution derive from? Why are statistical mechanics seemingly at the heart of all these simulation systems? Why is simulated annealing such a powerful metaphor in computational modelling techniques? Why is there a profound relationship between heat and entropy and why does that concordance appear to be useful - essential, even - for driving something so trivial as a system that predicts urban legend propagation? My intuition suggests to me that the answer to these questions is actually contained in M-theory - specifically the Hamiltonian - but, at the risk of completely derailing this discussion, I have to leave this as a future direction.
} 
tions are required in order to obtain a statistically significant difference between estimates obtained from separate theories, the effect is not as strong.

I am particularly interested in this possibility because I believe it offers something translatable directly into psychological science in a form that is immediately recognizable. ${ }^{22}$ Once likelihoods are attached to simulation results, permitting quantifiable tests to contrast between empirically-calibrated models versus null models, I believe Agent-Based Models could become first-class research methods within psychological science.

\subsection{A General Method for Psychological Research}

Altogether, the current work presents a general method for psychological research. This style of computational modelling, which is a synthesis of classic agent-based modelling and the epistemology of computational ecology, can be applied across the psychology literature. The class of regression models contains thousands of existing examples of psychological phenomena - and many of these models can be adapted and calibrated to use this approach. I would also expect this approach to work with many other behavioural frameworks, including those that are not described as linear models.

Consider the following general three-step program of study.

1. A highly-controlled, small-scale laboratory study is conducted.

2. A computational model is constructed based on the lab results.

3. The computational model is adapted to conditions that exist outside the laboratory.

In this manner, both strict experimental controls and ecological validity can be obtained.

\section{Conclusion}

\subsection{Adapting Linear Models to Agent-Based Modelling}

If the methods from this work can be generalized, then almost any psychological regression model can be translated to a computational model. The general epistemology developed by ecologists for use with plant and animal populations would appear to work with human phenomena as well.

The current work demonstrated the entire computational modelling process using a study of urban legends. The Eriksson and Coultas model of urban legend transmission did not produce viral outcomes in their laboratory study. When the same model was transplanted, unmodified, into a more plausible network context, high-disgust stories were transmitted at an increasing rate over time, just as expected.

\footnotetext{
${ }^{22}$ For example, this approach could be reminiscent of bootstrapping.
} 
This work demonstrates that a specific empirical finding can be "reverse engineered" from the literature and examined in new ways. The laboratory provides an ideal environment for highly-controlled research, which comes at the expense of ecological validity. Computational methods permit the laboratory results to be examined in a more ecologically valid context.

\subsection{Urban Legends Require Networks}

In order for a story to truly become prolific within a population, it must have many opportunities to be retold. When the community is structured so as to minimize the number of times stories are told, it causes an already-unlikely storytelling event to become diminished towards nothingness. The inverse can also be inferred: when stories are told to large audiences, the likelihood of retelling is increased as a simple function of the audience size.

Online social networks, in which a single account may have millions of connections, provide the opportunity for individuals to share stories widely. Urban legends are told as if they are true, entirely irrespective of whether or not they actually are true, which manifests in the present-day context as misinformation. Network topologies, on their own, are likely a major contributor to the proliferation of misinformation.

In the absence of modern online social networks, misinformation would likely remain an expensive proposition that could only be accomplished by wealthy actors like governments. The reduced cost of engaging in storytelling episodes has minimized the expenses associated with misinformation. Stories that are practically unbelievable have a chance at becoming widespread, as a simple function of network topology.

Even as the cost to spread urban legends is reduced, the cost for high-quality information remains high. For example, within the scientific community, open access journals are rare and the widespread availability of facts more closely resembles a serial reproduction task than an online social network. These differing network topologies imply troubling consequences for stories that propagate the truth, as opposed to those that spread lies.

In the end of the day, emotional selection would appear to interact with network topology in a meaningful way to determine which stories become prolific - but it appears that the network is ultimately the more influential factor. 


\section{References}

Allport, G. W., \& Postman, L. (1946). An Analysis of Rumor. Public Opinion Quarterly, 10(4), 501-517. doi: 10.1086/265813

Asch, S. E. (1955). Opinions and social pressure. Scientific American, 193(5), $31-35$.

Bankes, S. C. (2002, May). Agent-based modeling: A revolution? Proceedings of the National Academy of Sciences, 99 (suppl 3), 7199-7200. doi: 10.1073/ pnas.072081299

Banks, T., Fischler, W., Shenker, S. H., \& Susskind, L. (1997, April). M Theory As A Matrix Model: A Conjecture. Physical Review D, 55(8), 5112-5128. doi: 10.1103/PhysRevD.55.5112

Barabási, A.-L., \& Albert, R. (1999). Emergence of scaling in random networks. science, 286(5439), 509-512.

Bartlett, F. C. (1932). Remembering: An experimental and social study. Cambridge: Cambridge University.

BBC Staff. (2006, November). Star Wars Kid is top viral video. $B B C$.

Berger, J. (2011). Arousal Increases Social Transmission of Information. Psychological Science, 22(7), 891-893.

Berger, J., \& Milkman, K. (2010). Social Transmission, Emotion, and the Virality of Online Content. Wharton Research Paper.

Berry, B. J. L., Kiel, L. D., \& Elliott, E. (2002, May). Adaptive agents, intelligence, and emergent human organization: Capturing complexity through agent-based modeling. Proceedings of the National Academy of Sciences, 99 (suppl 3), 7187-7188. doi: 10.1073/pnas.092078899

Best, J., \& Horiuchi, G. T. (1985). The Razor Blade in the Apple: The Social Construction of Urban Legends. Social Problems, 32(5), 488-499.

Bikhchandani, S., Hirshleifer, D., \& Welch, I. (1998). Learning from the behavior of others: Conformity, fads, and informational cascades. Journal of economic perspectives, 12(3), 151-170.

Blackmore, S. (1998). Imitation and the definition of a meme. Journal of Memetics.

Blackmore, S. (1999). The meme machine. Oxford [England] ; New York: Oxford University Press. (11th floor robarts)

Blackmore, S. (2000). The Power Of Memes.. 
Bonabeau, E. (2002). Agent-based modeling: Methods and techniques for simulating human systems. Proceedings of the National Academy of Sciences, 99 (suppl 3), 7280-7287.

Boyd, R., \& Richerson, P. J. (2000). Meme theory oversimplifies cultural change. Scientific American, 283, 54-55.

Boyd, R., Richerson, P. J., \& Henrich, J. (2013). The cultural evolution of technology: Facts and theories. Cultural evolution: society, technology, language, and religion, 119-142.

Butler, D. (2009, April). Swine flu goes global. Nature, 458(7242), 1082-1083. doi: $10.1038 / 4581082 \mathrm{a}$

Campbell, D. T. (1957, July). Factors relevant to the validity of experiments in social settings. Psychological Bulletin, 54(4), 297-312. doi: http://dx.doi .org.myaccess.library.utoronto.ca/10.1037/h0040950

Centola, D., \& Macy, M. (2007). Complex Contagions and the Weakness of Long Ties. American Journal of Sociology, 113(3), 702-734.

Charisi, V., Dennis, L., Fisher, M., Lieck, R., Matthias, A., Slavkovik, M., ... Yampolskiy, R. (2017, March). Towards Moral Autonomous Systems. arXiv:1703.04741 [cs].

Chattoe-Brown, E. (2009, December). The social transmission of choice: A simulation with applications to hegemonic discourse. Mind 83 Society, 8(2), 193-207.

Cheng, J., Kleinberg, J., Leskovec, J., Liben-Nowell, D., Subbian, K., \& Adamic, L. (2018). Do Diffusion Protocols Govern Cascade Growth? In Twelfth International AAAI Conference on Web and Social Media.

Conway, J. H. (1976). On numbers and games. New York: Academic Press.

Dawkins, R. (1976). The selfish gene. New York: Oxford University Press.

Dawkins, R. (1993). Viruses of the mind. Dennett and his critics: Demystifying mind, 13-27.

Dennett, D. (1990). Memes and the exploitation of imagination. Journal of aesthetics and art criticism, 127-135.

De Waal, F. B. M. (2008). Putting the Altruism Back into Altruism: The Evolution of Empathy. Annual Review of Psychology, 59, 279-300.

Dichter, E. (1966). How Word-of-Mouth Advertising Works. Harvard Business Review, $44(6), 147$.

Doran, D., Schulz, S., \& Besold, T. R. (2017, October). What Does Explainable AI Really Mean? A New Conceptualization of Perspectives. arXiv:1710.00794 [cs]. 
Dreyer, J. L. E. J. L. E. (1906). History of the planetary systems from Thales to Kepler. Cambridge University Press.

Dublin, L. I., \& Lotka, A. J. (1925). On the true rate of natural increase: As exemplified by the population of the United States, 1920. Journal of the American statistical association, 20(151), 305-339.

Dundes, A. (1965). The Study of Folklore in Literature and Culture: Identification and Interpretation. The Journal of American Folklore, 78(308), 136-142. doi: $10.2307 / 538280$

Edwards, D., \& Middleton, D. (1990). Collective remembering. Sage.

Epstein, J. M. (1999). Agent-based computational models and generative social science. , 20.

Epstein, J. M. (2006). Generative Social Science: Studies in agent-based computational modeling. Princeton University Press.

Epstein, J. M. (2014). Agent_Zero: Toward Neurocognitive Foundations for Generative Social Science. Princeton: Princeton University Press.

Epstein, J. M., \& Axtell, R. L. (1996). Growing Artificial Societies: Social Science from the Bottom Up. Brookings Institution Press.

Eriksson, K., \& Coultas, J. C. (2014). Corpses, Maggots, Poodles and Rats: Emotional Selection Operating in three Phases of Cultural Transmission of Urban Legends. Journal of Cognition and Culture, 14(1-2), 1-26.

Euler, L. (1953). Leonhard Euler and the Koenigsberg Bridges. Scientific American, 189(1), 66-72.

Fowler, J. H., \& Christakis, N. A. (2010). Cooperative Behavior Cascades in Human Social Networks. Proceedings of the National Academy of Sciences, 107(12), 5334-5338.

Freedman, J. L., \& Perlick, D. (1979, May). Crowding, contagion, and laughter. Journal of Experimental Social Psychology, 15(3), 295-303. doi: 10.1016/ 0022-1031(79)90040-4

Friggeri, A., Adamic, L. A., Eckles, D., \& Cheng, J. (2014). Rumor Cascades. In ICWSM.

García, A. P., \& Rodríguez-Patón, A. (2016, April). Analyzing Repast Symphony models in R with RRepast package. bioRxiv, 047985. doi: 10.1101/ 047985

Gelman, A., \& Shalizi, C. R. (2013). Philosophy and the practice of Bayesian statistics. British Journal of Mathematical and Statistical Psychology, 66(1), $8-38$. 
Goffman, W., \& Newill, V. A. (1964, October). Generalization of Epidemic Theory: An Application to the Transmission of Ideas. Nature, 204(4955), 225-228. doi: $10.1038 / 204225 \mathrm{a} 0$

Grabowski, A., \& Kosinski, R. A. (2005). The SIS model of epidemic spreading in a hierarchical social network. Acta Physica Polonica B, 36(5), 1579-1593.

Granovetter, M. S. (1973). The Strength of Weak Ties. American Journal of Sociology, 78(6), 1360-1380.

Grimm, V., Berger, U., Bastiansen, F., Eliassen, S., Ginot, V., Giske, J., ... DeAngelis, D. L. (2006, September). A standard protocol for describing individual-based and agent-based models. Ecological Modelling, 198(1-2), 115-126. doi: 10.1016/j.ecolmodel.2006.04.023

Grimm, V., Berger, U., DeAngelis, D. L., Polhill, J. G., Giske, J., \& Railsback, S. F. (2010, November). The ODD protocol: A review and first update. Ecological Modelling, 221(23), 2760-2768. doi: 10.1016/j.ecolmodel.2010.08 .019

Grimm, V., \& Railsback, S. F. (2005). Individual-based modeling and ecology (Vol. 2005). BioOne.

Hatfield, E., Cacioppo, J. T., \& Rapson, R. L. (1993). Emotional contagion. Current Directions in Psychological Science, 2(3), 96-99. doi: 10.1111/1467 -8721.ep10770953

Heath, C., Bell, C., \& Sternberg, E. (2001). Emotional Selection in Memes: The Case of Urban Legends. Journal of Personality and Social Psychology, $81(6), 1028$.

Hethcote, H. W. (1974). Asymptotic Behavior and Stability in Epidemic Models. In P. van den Driessche (Ed.), Mathematical Problems in Biology (p. 83-92). Springer Berlin Heidelberg.

Hethcote, H. W. (1994). A Thousand and One Epidemic Models. In S. A. Levin (Ed.), Frontiers in Mathematical Biology (p. 504-515). Springer Berlin Heidelberg.

Heyer, P. (2003). America under attack I: A reassessment of Orson Welles' 1938 war of the worlds broadcast. Canadian Journal of Communication; Toronto, 28(2), 149-165.

Hodas, N. O., \& Lerman, K. (2014). The simple rules of social contagion. Scientific reports, 4, 4343.

Jaynes, E. T. (1957). Information theory and statistical mechanics. Physical review, $106(4), 620$. 
Jennings, N. R., Sycara, K., \& Wooldridge, M. (1998, January). A Roadmap of Agent Research and Development. Autonomous Agents and Multi-Agent Systems, 1(1), 7-38. doi: 10.1023/A:1010090405266

Jiang, L., Miao, Y., Yang, Y., Lan, Z., \& Hauptmann, A. G. (2014). Viral Video Style: A Closer Look at Viral Videos on YouTube. In Proceedings of International Conference on Multimedia Retrieval (pp. 193:193-193:200). New York, NY, USA: ACM. doi: 10.1145/2578726.2578754

Johnson, D. M. (1945). The "Phantom Anesthetist" of Mattoon: A Field Study of Mass Hysteria. The Journal of Abnormal and Social Psychology, 40(2), 175-186. doi: 10.1037/h0062339

Kermack, W. O., \& McKendrick, A. G. (1927, January). A Contribution to the Mathematical Theory of Epidemics. Proceedings of the Royal Society of London. Series A, 115(772), 700-721. doi: 10.1098/rspa.1927.0118

Kyanka, R. (1999, November). Something Awful.

Le Bon, G. (1895). The Crowd. Routledge. doi: 10.4324/9781315131566

Leskovec, J., McGlohon, M., Faloutsos, C., Glance, N., \& Hurst, M. (2007, April). Cascading Behavior in Large Blog Graphs. arXiv:0704.2803 [physics].

Liu, J., \& Ashton, P. S. (1995). Individual-based simulation models for forest succession and management. Forest Ecology and Management, 73(1-3), 157175 .

Lohmann, S. (1994). The dynamics of informational cascades: The Monday demonstrations in Leipzig, East Germany, 1989-91. World politics, 47(01), $42-101$.

Lotan, G., Graeff, E., Ananny, M., Gaffney, D., Pearce, I., \& m. boyd, d. (2011). The Arab Spring - The Revolutions Were Tweeted: Information Flows During the 2011 Tunisian and Egyptian Revolutions. International Journal of Communication, 5, 31 .

Luke, S., Cioffi-Revilla, C., Panait, L., Sullivan, K., \& Balan, G. (2005, January). MASON: A Multiagent Simulation Environment. SIMULATION, 81(7), 517-527. doi: 10.1177/0037549705058073

Macy, M. W., \& Willer, R. (2002). From Factors to Actors: Computational Sociology and Agent-Based Modeling. Annual Review of Sociology, 28(1), 143-166. doi: 10.1146/annurev.soc.28.110601.141117

Miller, I. D. (2012). The Social Transmission of User-Generated Memes (MA Thesis, University of Toronto, Toronto, ON, Canada). doi: 1807/67214

Mullen, P. B. (1972). Modern Legend and Rumor Theory. Journal of the Folklore Institute, 9(2/3), 95-109. doi: 10.2307/3814160 
Nakagawa, E., \& Unebasami, K. (2007, January). I Can Has Cheezburger.

Nicol, C. J. (1995, September). The social transmission of information and behaviour. Applied Animal Behaviour Science, 44(2-4), 79-98.

Platt, J. R. (1964). Strong Inference. Science, 146 (3642), 347-353.

Pope, J. (1804). Description of an Orrery of His Construction. Memoirs of the American Academy of Arts and Sciences, 2(2), 43-45. doi: 10.2307/27670809

Railsback, S. F., \& Grimm, V. (2011). Agent-based and individual-based modeling: A practical introduction. Princeton university press.

Railsback, S. F., \& Harvey, B. C. (2002). Analysis of habitat-selection rules using anindividual-based model. Ecology, 83(7), 1817-1830.

Reid, T. (1832). Treatise on Clock and Watch Making: Theoretical and Practical. Carey and Lea.

Roediger, H. L., Meade, M. L., \& Bergman, E. T. (2001). Social contagion of memory. Psychonomic Bulletin \& Review, 8(2), 365-371.

Rogers, E. M. (1962). Diffusion of innovations. New York: Free Press of Glencoe.

SAclopedia. (2004, December). Image Macro [SAclopedia].

Schank, J. C. (2001, January). Beyond reductionism: Refocusing on the individual with individual-based modeling. Complexity, 6(3), 33-40. doi: 10.1002/cplx. 1026

Schelling, T. C. (1971). Dynamic models of segregation. Journal of Mathematical Sociology, 1(2), 143-186.

Schmuckler, M. A. (2001). What Is Ecological Validity? A Dimensional Analysis. Infancy, 2(4), 419-436. doi: 10.1207/S15327078IN0204_02

Schnall, S., Haidt, J., Clore, G. L., \& Jordan, A. H. (2008, August). Disgust as Embodied Moral Judgment. Personality and Social Psychology Bulletin, 34 (8), 1096-1109. doi: 10.1177/0146167208317771

Shannon, C. E. (1948, July). A Mathematical Theory of Communication. The Bell System Technical Journal, 27, 379-423.

Stubbersfield, J. M., Tehrani, J. J., \& Flynn, E. G. (2015). Serial Killers, Spiders and Cybersex: Social and Survival Information Bias in the Transmission of Urban Legends. British Journal of Psychology, 106 (2), 288-307.

Swarm Development Group. (2016, July). SWARMFEST 2016: 20th Annual Meeting on Agent Based Modeling $\&$ Simulation (Tech. Rep.). Burlington, Vermont: University of Vermont. 
Tedeschi, G., Mazloumian, A., Gallegati, M., \& Helbing, D. (2012). Bankruptcy cascades in interbank markets. PloS one, 7(12), e52749.

Tesfatsion, L. (2003, February). Agent-based computational economics: Modeling economies as complex adaptive systems. Information Sciences, $149(4)$, 262-268. doi: 10.1016/S0020-0255(02)00280-3

Thiele, J. C., Kurth, W., \& Grimm, V. (2014). Facilitating parameter estimation and sensitivity analysis of agent-based models: A cookbook using NetLogo and R. Journal of Artificial Societies and Social Simulation, 17(3), 11.

VanArsdale, D. W. (1998). Chain Letter Evolution.

Vickery, J. R. (2014, March). The curious case of Confession Bear: The reappropriation of online macro-image memes. Information, Communication $\mathcal{E}^{3}$ Society, 17(3), 301-325. doi: 10.1080/1369118X.2013.871056

von Neumann, J. (1966). Theory of self-reproducing automata (A. W. Burks, Ed.). Urbana: University of Illinois Press.

Walras, L. (1874). Elements of Pure Economics. Routledge. doi: 10.4324/ 9781315888958

Whiten, A., Spiteri, A., Horner, V., Bonnie, K. E., Lambeth, S. P., Schapiro, S. J., \& De Waal, F. B. M. (2007). Transmission of multiple traditions within and between chimpanzee groups. Current Biology, 17(12), 1038-1043.

Wilensky, U. (1999). NetLogo. Center for Connected Learning and ComputerBased Modeling, Northwestern University. Evanston, IL.

Wilensky, U., \& Rand, W. (2015). An introduction to agent-based modeling: Modeling natural, social, and engineered complex systems with NetLogo. MIT Press.

Wojnicki, A. C., \& Godes, D. (2008, April). Word-of-Mouth as SelfEnhancement. SSRN eLibrary. 


\section{Build Information}

1f341826f5b3043b1dd81c988fbdd52cf09f 154c

Thu Oct 10 16:20:10 UTC 2019 